\title{
Cable Tension Preslack Method Construction Simulation and Engineering Application for a Prestressed Suspended Dome
}

\author{
Xuechun Liu, ${ }^{1,2}$ Ailin Zhang, ${ }^{1,2}$ and Wanlin Fu ${ }^{1,2}$ \\ ${ }^{1}$ Beijing Engineering Research Center of High-Rise and Large-Span Prestressed Steel Structure, Beijing University of Technology, \\ Beijing 100024, China \\ ${ }^{2}$ College of Architecture and Civil Engineering, Beijing University of Technology, Beijing 100124, China
}

Correspondence should be addressed to Xuechun Liu; liuxuechun@bjut.edu.cn

Received 22 January 2015; Accepted 3 April 2015

Academic Editor: Antônio G. B. de Lima

Copyright (C) 2015 Xuechun Liu et al. This is an open access article distributed under the Creative Commons Attribution License, which permits unrestricted use, distribution, and reproduction in any medium, provided the original work is properly cited.

\begin{abstract}
To solve the shortage of traditional construction simulation methods for suspended dome structures, based on friction elements, node coupling technology, and local cooling, the cable tension preslack method is proposed in this paper, which is suitable for the whole process construction simulation of a suspended dome. This method was used to simulate the construction process of a largespan suspended dome case study. The effects on the simulation results of location deviation of joints, construction temperature, construction temporary supports, and friction of the cable-support joints were analyzed. The cable tension preslack method was demonstrated by comparing the data from the construction simulation with measured results, providing the control cable tension and the control standards for construction acceptance. The analysis demonstrated that the position deviation of the joint has little effect on the control value; the construction temperature and the friction of the cable-support joint significantly affect the control cable tension. The construction temperature, the temporary construction supports, and the friction of the cable-support joints all affect the internal force and deflection in the tensioned state but do not significantly affect the structural bearing characteristics at the load state. The forces should be primarily controlled in tensioned construction, while the deflections are controlled secondarily.
\end{abstract}

\section{Introduction}

Suspended dome structures are a type of new hybrid prestressed steel structure that consists of an upper rigid single layer latticed shell and a lower flexible cable-support system $[1,2]$. The stiffness of the upper latticed shell is small and the lower cable-support system has no stiffness before the structure's shape is formed. Therefore, its load-carrying systems are completely different before and after the shape is formed and are even constantly changing during the tensioning process. Although there is only self-weight during the construction, the span of a large-span suspended dome is so large that studying the safety in each state during the tensioning process is necessary. Full house scaffolds are generally used for construction of the upper latticed shell. For safety in cable tensioning, the temporary supports are normally not removed during the construction, which increases the difficulty of tensioning control [3]. Because the control cable tension is obtained by construction simulation and the construction acceptance is based on the simulation results, the effect of temporary supports must be considered.

Some scholars and engineers have simulated the construction of suspended domes and obtained some achievements [4]. Some useful construction simulation methods for suspended domes have been proposed: $\mathrm{Li}$ et al. proposed the cycle-approach method [5], Yuan and Dong proposed the inverse analysis of the construction process method [6], Zhang et al. carried out construction process simulation of a prestressed suspended dome by the birth-death element method [7], and Zhuo proposed the tension compensation method [8]. Wang et al. carried out a whole process tracing analysis of an oval suspended dome structure during shaping and analyzed structural deformation and variation of the internal forces during the tensioning process $[9,10]$. Zhang et al. did the numerical simulation of the 2008 Olympic Games badminton gymnasium suspended dome structure [11]. Guo [12] and Nie et al. [13] carried out construction simulations and scaled model tests on the suspended dome 
structure based on engineering. However, the effects on the internal force and displacement of the structure are not simultaneously considered in these traditional methods, including the location deviation of the joint, construction temporary supports, construction temperature, friction of the cable-support joint, local tension of the cable, and other factors, so the above methods did not simulate the actual construction process very accurately.

A new method of construction simulation, the cable tension preslack method, is proposed in this paper to solve this problem. This method was used to simulate the construction process of a large-span suspended dome and the results of the construction simulation were used for the tensioning control value. The simulation value and field measured value were compared to study the effects of the location deviation of the joint, construction temporary supports, construction temperature, friction of the cable-support joint, and local tension of the cable.

\section{Method of Construction Simulation}

Currently, the traditional method used in engineering design is the once loading method, where the external load and initial cable strain are applied once to the structure according to a reasonable load substep, which is inaccurate because the effect of the construction process on the final stress of the structure is not considered. The tensioning sequence, cable tension, and friction force greatly affect the stress of the structure. At present, the construction simulation methods applied to practical engineering include the once loading method, the birth-death element method, the tension relaxation method, and the tension compensation method.

The once loading method is conventionally used in engineering design to apply the load once to the calculation model and to analyze the structure without considering the effect of the loading process. Thus the calculation is not strict according to the construction process. When a nonconservative force acts or the structure enters into an elastic-plastic state, the calculation result is different from the actual construction.

The entire model is built once in the birth-death element method, and the entire construction process is simulated by the birth or death of elements and continuous analysis based on multiple time steps. When the outermost hoop is tensioned, the cable elements of the other hoops are in a death state. At this time, the death elements remain deforming with the deformation of the structure, but the weight is zero and the stiffness is almost zero, so they have no effect on the structure. The hoop cables are tensioned from outmost loop to innermost loop by activating relevant elements one loop by one loop until all hoop cables are activated. Let $l$ be the initial length of the cable element and let $l \varepsilon A_{c a}$ be the design initial cable force due to the deformation compatibility. When the element is activated, the length becomes $l+l_{\Delta}$, the initial cable force is $\left(l+l_{\Delta}\right) \varepsilon A_{c a}$, and the difference between the actual cable force and the design cable force is $l_{\Delta} \varepsilon A_{c a}$. In addition, the untensioned elements are killed in the birthdeath element method and the killed elements have no weight. However, all the cables are always put in place in actual construction in the relaxed state so that the weight has been applied to the structure, so the effect of the cable selfweight is ignored in the birth-death element method.

The tension relaxation method analyzes the tensioning process by inversing the construction sequence. It assumes that the first analysis state is that all the cables are tensioned to their design value, and then the cables are relaxed according to the inverse sequence of cable tensioning. The control values of the cable tension at each sequence are calculated one by one during the relaxation. The greatest feature of the tension relaxation method is that multiple cycles of calculations are not needed. However, the state in which all the cables are completely tensioned must be obtained first, and this state has to be achieved in a finite element model. In fact, the construction finished state needs to be determined by other methods before the control value of the cable tension can be derived, making this method very difficult to be applied in practice. Moreover, when friction exists in the cablesupport joint, the magnitude and direction of the friction are constantly changing during the tensioning process, and the construction process is irreversible, so the real construction process cannot be simulated by this method.

The tension compensation method constantly adjusts the cable force by tension compensation based on the actual internal forces of the cable in equilibrium making it approach to the force in equilibrium state gradually. This method uses gradual iterative approximation, for which many rounds of iterative calculations are needed. The calculation of this method does not completely follow the steps of the construction process. When friction forces exist in the cablesupport joint, the magnitude and direction of the friction are constantly changing during the tensioning process, meaning that the real change of friction cannot be simulated by this method and the entire construction process cannot be simulated accurately.

Because the actual construction process cannot be simulated by the above methods, a new construction simulation method, the cable tension preslack method, is proposed in this paper. The hoop cables are not tensioned at each segment in the actual construction; instead, several tensioning points are established and the hoop cables are only tensioned at each tensioning point. The tensioning control value at each tensioning point is calculated based on the unchanged total elongation of one hoop cable. The total strain of one hoop cable is calculated according to the design initial strain of the cable, and then the total strain is transformed into negative temperature and the average is assigned to each tensioning point. The segments for each hoop cable have different cable forces from the friction in the cable-support joint; the cable tension is greater than the design value at the tensioning point and less than the design value away from the tensioning point. The initial strains of all the cables were set to zero before being tensioned, and large heating loads were applied to all cables to keep them in a relaxed state until they were tensioned. Then, according to the construction steps, the negative temperatures were applied to each tensioning point. Each tensile state simulated by the cable tension preslack method is the same as that of the actual structure, and the operation is simple for engineers to understand and apply. 
TABLE 1: Comparison of different tensioning methods.

\begin{tabular}{lccc}
\hline Influence factors & & Tensioning methods & \\
& Radial cables tensioning & Struts elongation & Hoop cables tensioning \\
\hline Number of jacks & Large & Medium & Small \\
Tensioning force & Small & Medium & Large \\
Prestress loss & Medium & Small & Large \\
Mutual effect between cables & Large & Medium & Small \\
Controllability of prestress loss & Hard & Medium & Simple \\
Controllability of synchronous tension & Hard & Medium & Simple \\
Construction period & Long & Medium & Short \\
Costs of construction & High & Medium & Low \\
Effect of temporary supports & Medium & Large & Small \\
\hline
\end{tabular}

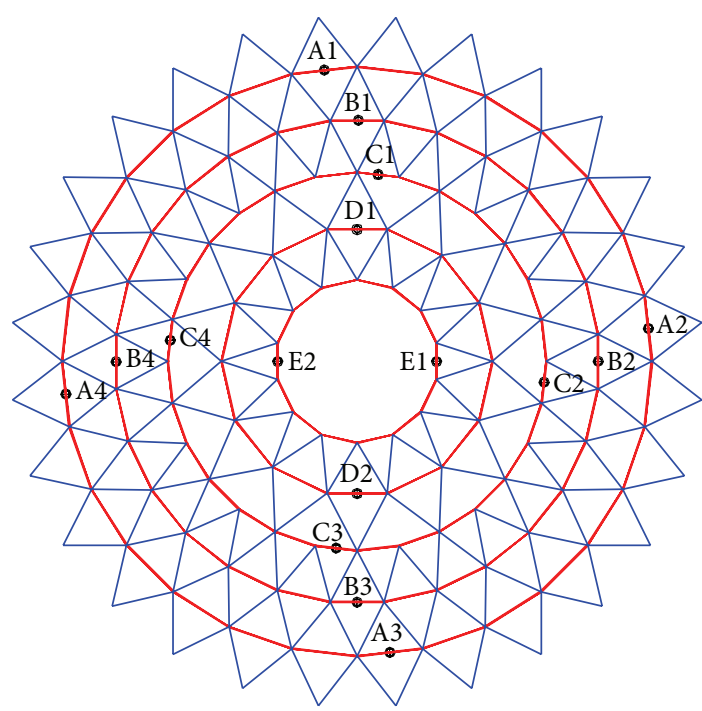

FIGURE 1: Sketch of tensioning points on hoop cables.

The method was verified by simulating the construction of a large-span suspended dome structure successfully.

\section{Tensioning Scheme}

Three methods are mainly used for prestressed applications: radial cables tensioning, struts elongation, and hoop cables tensioning. Many factors including the number of tensioning points and jacks, the magnitude of tension and prestress loss, the mutual effect between cables, the controllability of prestress loss and synchronous tensioning, the construction period, and the costs of construction were considered for the selection of the tensioning method. Through the comparisons shown in Table 1, the hoop cables tensioning was selected.

To reduce the effect of friction on the cable-strut joint, two or four tensioning points were set on each cable hoop, as shown in Figure 1. Taking into account the number of cablestrut joints and jacks, the synchronous tensioning, the construction period, the cost of construction, the magnitude of the tensioning force, and prestress loss control, the following tensioning sequences were selected. The 5 hoop cables were tensioned in two stages. Each cable was tensioned to $70 \%$ of the design value in the first stage following the sequence $\mathrm{A} \rightarrow \mathrm{B} \rightarrow \mathrm{C} \rightarrow \mathrm{D} \rightarrow \mathrm{E}$ before all the temporary supports were removed; each cable was then tensioned to $110 \%$ of the design value and offloaded to $100 \%$ in the second stage following the sequence $\mathrm{A} \rightarrow \mathrm{B} \rightarrow \mathrm{C} \rightarrow \mathrm{D} \rightarrow \mathrm{E}$ to reduce friction effects. As shown in Table 2 , the design values of hoop cables are initial strains which are got by the optimization and design at the stage of construction drawing design and preliminary design. And then, they are inverted to negative temperature to be applied on the tensioning point in analysis model according to the tensioning sequence. The model is analyzed and the tensioning force of each tensioning point is got for each state during the tensioning sequence.

The tensioning force of each state is applied on the tensioning point which is set in the real structure as shown in Figure 2. When the jack contracts, the tension force will occur in the steel bar, the distance between the two clamps will shorten, at the same time, turning the rifled tube, making the turnbuckles being screwed into the rifled tube, and then the hoop cable will shorten; in this way, the hoop cable is tensioned. After tensioning, the jack and clamp are taken off and then the tension force in the steel bar is converted to the turnbuckle and rifled tube.

\section{Shape-Finding Methods of Suspended Dome}

The shape of a suspended dome changes constantly during the tensioning process with three main states: zero state, tensioned state, and load state. The zero state is the installation state before the structure is tensioned and loaded. The structure arches to the tensioned state after being tensioned, and thereafter all the dead loads are applied, making the tensioned state become the load state. The shape-finding of a suspended dome is performed as follows: first, the design state is taken as the zero state, the model is built, and the dead loads are applied; then the joint location is obtained by mechanical analysis. The difference in the calculated joints location with those of the zero state is determined, and then the joint location is revised by the difference to form a new state. The mechanical analysis is performed again, and the joint location is revised again until the joint locations are the same as the design locations. Actually, the suspended dome has 


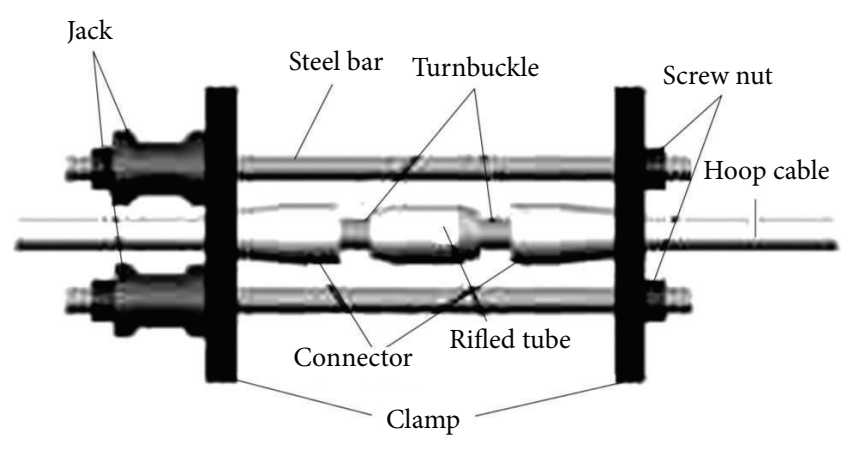

(a) Tensioning setup sketch

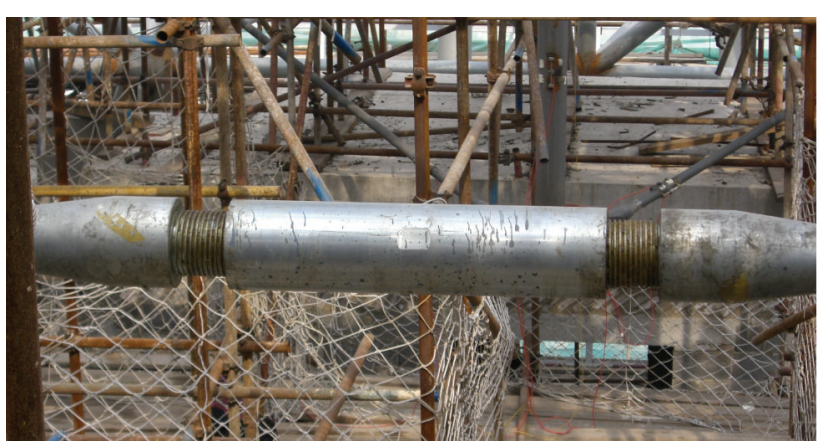

(b) Picture of tensioning point

Figure 2: Tensioning points on hoop cables.

TABLE 2: The design initial strain of tensioning point.

\begin{tabular}{lcccc}
\hline Number of hoop cables & $\begin{array}{c}\text { Design initial strain } \\
\text { of hoop cables }\end{array}$ & $\begin{array}{c}\text { Coefficient of linear } \\
\text { expansion } 1 /{ }^{\circ} \mathrm{C}\end{array}$ & $\begin{array}{c}\text { Negative temperature } \\
\text { of tensioning point }{ }^{\circ} \mathrm{C}\end{array}$ & $\begin{array}{c}\text { Number of tensioning } \\
\text { point }\end{array}$ \\
\hline 1 & 0.00261 & $1.20 E-05$ & 1523 & 4 \\
2 & 0.00339 & $1.20 E-05$ & 1974 & 4 \\
3 & 0.00269 & $1.20 E-05$ & 1567 & 4 \\
4 & 0.00323 & $1.20 E-05$ & 1882 & 2 \\
5 & 0.00250 & $1.20 E-05$ & 1460 & 2 \\
\hline
\end{tabular}

some stiffness before being shaped, and the deformation by self-weight is smaller. The stiffness becomes larger after being shaped so it can meet the deformation code requirement for the design of steel structures [14]. Therefore, a suspended dome structure, similar to an ordinary steel structure, does not need the shape-finding analysis.

\section{Construction Temporary Supports}

The full house scaffold is used in construction for the upper latticed shell; all joints, components, and supports that are installed depend on the scaffold. During the lifting and welding of the upper latticed shell, temporary supports are used to support the structural self-weight and ensure accurate positioning and welding quality. To further reduce the risk in tensioning, temporary supports are not fully dismantled. The layouts of temporary supports are shown in Figure 3, where one point represent one support.

Many factors, including the stiffness, the initial state, and contact state with joints and the status variation of temporary supports in the tensioning process, must be considered for the construction simulation. As shown in Figure 4, circular hollow tubes were used for supports on the eight $20 \mathrm{~m}$ high scaffolds on the ground. Because temporary supports can only bear compression but not tension, the COMBIN39 element in ANSYS was used to simulate the temporary supports. The temporary supports are out of action after the joints separate from the temporary supports, because they were removed once separate. The initial pressure of the temporary supports is supposed to be zero before they bear the self-weight of the shell, not considering the stress

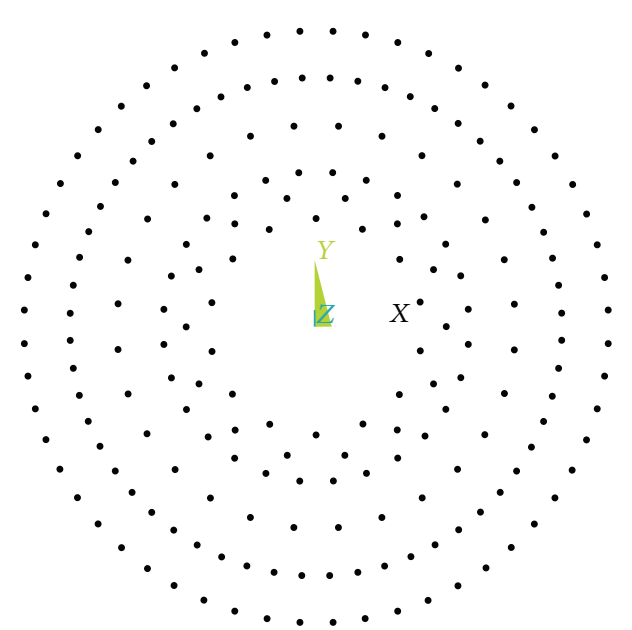

FIGURE 3: Layout of temporary supports.

in the temporary support introduced by welding stress and construction forces in the construction process. The stiffness of the temporary support is

$$
K=\frac{E n s}{l}
$$

$E$ is the elastic modulus of steel;

$n$ is the number of scaffolds;

$s$ is the area of a single scaffold;

$l$ is the length of scaffold. 


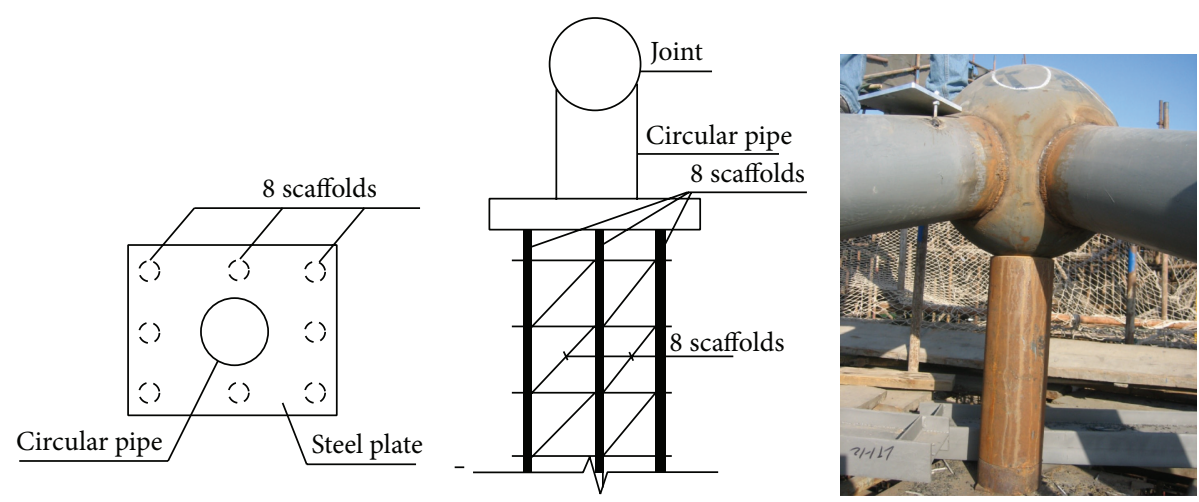

FIGURE 4: Temporary supports.

TABLE 3: Axial force of cable-support system at tensioned state [kN].

\begin{tabular}{|c|c|c|c|c|c|c|c|c|}
\hline \multirow{3}{*}{$\begin{array}{l}\text { Cable-support system number } \\
\text { 1st hoop }\end{array}$} & \multicolumn{4}{|c|}{ Original design force } & \multicolumn{4}{|c|}{ Force considering position deviation } \\
\hline & \multirow{2}{*}{$\begin{array}{r}\text { Strut } \\
-181\end{array}$} & \multicolumn{2}{|c|}{ Radial rod } & \multirow{2}{*}{$\begin{array}{c}\text { Hoop cable } \\
2828\end{array}$} & \multirow{2}{*}{$\begin{array}{l}\text { Strut } \\
-181\end{array}$} & \multicolumn{2}{|c|}{ Radial rod } & \multirow{2}{*}{$\frac{\text { Hoop cable }}{2837}$} \\
\hline & & 411 & 411 & & & 412 & 412 & \\
\hline 2nd hoop & -95 & 179 & 179 & 1293 & -95 & 179 & 179 & 1291 \\
\hline 3rd hoop & -70 & 110 & 110 & 833 & -70 & 111 & 111 & 833 \\
\hline 4th hoop & -84 & 131 & 131 & 425 & -84 & 132 & 132 & 426 \\
\hline 5th hoop & -47 & 65 & 65 & 230 & -47 & 65 & 65 & 230 \\
\hline
\end{tabular}

TABLE 4: Axial force of cable-support systems in construction simulation [kN].

\begin{tabular}{|c|c|c|c|c|c|c|c|c|}
\hline \multirow{3}{*}{$\begin{array}{l}\text { Cable-support system number } \\
\text { 1st hoop }\end{array}$} & \multicolumn{4}{|c|}{ Tensioned state } & \multicolumn{4}{|c|}{ Design state } \\
\hline & \multirow{2}{*}{$\begin{array}{l}\text { Strut } \\
-197\end{array}$} & \multicolumn{2}{|c|}{ Radial rod } & \multirow{2}{*}{$\frac{\text { Hoop cable }}{3048}$} & \multirow{2}{*}{$\begin{array}{l}\text { Strut } \\
-179\end{array}$} & \multicolumn{2}{|c|}{ Radial rod } & \multirow{2}{*}{$\begin{array}{c}\text { Hoop cable } \\
2804\end{array}$} \\
\hline & & 443 & 443 & & & 407 & 407 & \\
\hline 2nd hoop & -95 & 177 & 177 & 1285 & -92 & 172 & 172 & 1250 \\
\hline 3rd hoop & -68 & 107 & 107 & 803 & -66 & 105 & 105 & 791 \\
\hline 4th hoop & -80 & 125 & 125 & 403 & -79 & 124 & 124 & 401 \\
\hline 5th hoop & -45 & 61 & 61 & 215 & -45 & 60 & 60 & 214 \\
\hline
\end{tabular}

\section{Influencing Factors Analysis}

6.1. Effects on Tensioned State. The axial forces of the cablesupport system at the tensioned state are shown in Table 3 according to the actual deviations measured on site. As shown in the table, the position deviations of the joints had little effect on the force of the cable-support system. The design axial forces of the strut and radial rod were the same as that considering joint deviation, and the difference in cable tension of the hoop cable was only $0.3 \%$, which can be neglected.

Because the construction of the upper layer shell was in the hot summer when the average temperature was $30^{\circ} \mathrm{C}$ and the tensioning was in the cold winter when the average temperature was $0^{\circ} \mathrm{C}$, the design reference temperature was $20^{\circ} \mathrm{C}$. The tensioned state at $0^{\circ} \mathrm{C}$ is not the design state; the design state occurs when the entire structure is heated to $20^{\circ} \mathrm{C}$ after being tensioned. The temperature of the upper latticed shell is therefore decreased by $30^{\circ} \mathrm{C}$ and that of the cable-support system is decreased by $20^{\circ} \mathrm{C}$ in the construction simulation. The axial forces of the cable-support system in the tensioned state and in the design state are shown in Table 4. As shown in the table, the tension of the first hoop cable at the tensioned state is larger than that of the original design; however, the tensions of the other cables are smaller than the design values (Table 3 ). The cable forces of the design state are similar to that of the original but not identical. Because the whole structural construction is not under the design reference temperature, there is still a temperature difference of $-10^{\circ} \mathrm{C}$ in the latticed shell at the design state. The entire structure cannot be heated to the design reference temperature simultaneously, but the temperature makes a $0.85-7.0 \%$ difference in the cable force. Therefore, the construction temperature has some effect on the cable force, so it must be considered in construction simulations.

According to the above calculation method for the stiffness of temporary supports, the stiffness of the temporary 
TABLE 5: Axial force of cable-support system at tensioned state considering temporary supports or friction of cable-support joint [kN].

\begin{tabular}{|c|c|c|c|c|c|c|c|c|}
\hline \multirow{3}{*}{$\begin{array}{l}\text { Cable-support system number } \\
\text { 1st hoop }\end{array}$} & \multicolumn{4}{|c|}{ Considering temporary supports } & \multicolumn{4}{|c|}{ Considering friction of cable-support joint } \\
\hline & \multirow{2}{*}{$\begin{array}{l}\text { Strut } \\
-177\end{array}$} & \multicolumn{2}{|c|}{ Radial rod } & \multirow{2}{*}{$\begin{array}{c}\text { Hoop cable } \\
2769\end{array}$} & \multirow{2}{*}{$\begin{array}{l}\text { Strut } \\
-180\end{array}$} & \multicolumn{2}{|c|}{ Radial rod } & \multirow{2}{*}{$\frac{\text { Hoop cable }}{2999}$} \\
\hline & & 402 & 402 & & & 508 & 345 & \\
\hline 2nd hoop & -100 & 186 & 186 & 1349 & -99 & 228 & 143 & 1370 \\
\hline 3rd hoop & -72 & 112 & 112 & 846 & -74 & 151 & 82 & 895 \\
\hline 4th hoop & -67 & 132 & 132 & 426 & -91 & 166 & 117 & 478 \\
\hline 5th hoop & -84 & 65 & 65 & 230 & -52 & 89 & 53 & 260 \\
\hline
\end{tabular}

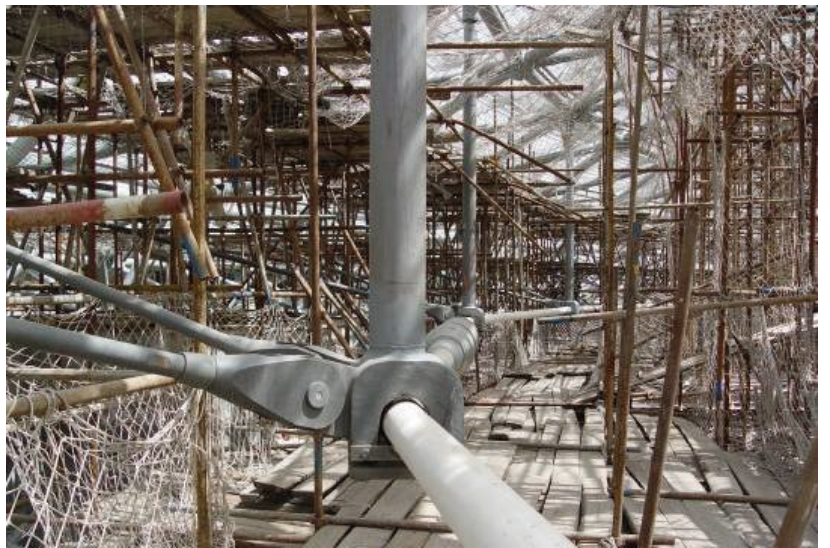

(a) Cable-support joint

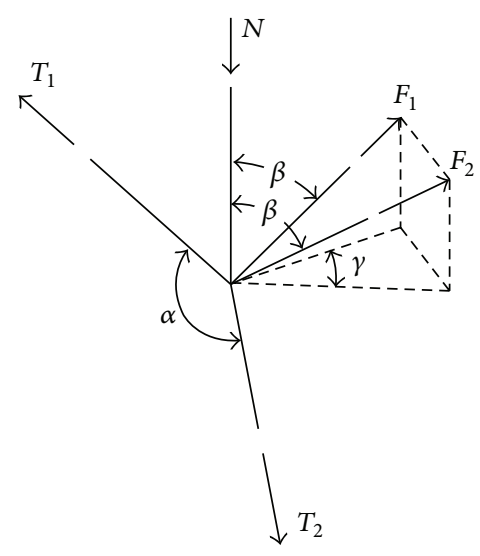

(b) Force equilibrium system

FIGURE 5: Sketch of the cable-support system.

supports on the outermost hoop is $80587 \mathrm{kN} / \mathrm{m}$ and the other stiffness is $40292 \mathrm{kN} / \mathrm{m}$. The tensioning simulation of the suspended dome with temporary supports was performed, and the axial forces of the cable-support system in the tensioned state are shown in Table 5. As shown in the table, the force of the first hoop cable is smaller than that of the design (shown in Table 3), the forces of the second and third hoop cables are larger than that of the design, and the forces of the fourth and fifth hoop cables are almost the same as that of the design. This is because that the first and second hoop's temporary supports are not separated from the structure after tensioning is finished, they still play supporting roles. In addition, the latticed shell could fully bear the weight of the structure and construction loads without temporary supports, so removing the temporary supports before tensioning is better selection.

The friction of the cable-support joints can only be obtained by field measurement because of fabrication and construction. As shown in Figure 5, two segments of hoop cable, two radial rods and one strut make up a force equilibrium system at each cable-support joint. The tensions of the radial rods were obtained by first setting vibratory strain gauges on the struts and steel rods. Next, the tension of the hoop cable, the pressure of the strut, and the friction coefficient were determined by the force equilibrium equation. The angle between hoop cables is $\alpha$, the angle between the steel rod and strut is $\beta$, the projecting angle of the steel rods in the horizontal plane is $\gamma$, the tensions of the hoop cables are $T_{1}$ and $T_{2}$, the forces of the radial steel $\operatorname{rod}$ are $F_{1}$ and $F_{2}$, the pressure of the strut is $N$, and the friction coefficient is $\mu$ :

$$
\begin{aligned}
T_{1}= & \frac{\left(F_{1}+F_{2}\right) \sin \beta \cos (\gamma / 2)}{2 \cos (\alpha / 2)} \\
& -\frac{\left(F_{1}-F_{2}\right) \sin \beta \sin (\gamma / 2)}{2 \sin (\alpha / 2)}, \\
T_{2}= & \frac{\left(F_{1}+F_{2}\right) \sin \beta \cos (\gamma / 2)}{2 \cos (\alpha / 2)} \\
& +\frac{\left(F_{1}-F_{2}\right) \sin \beta \sin (\gamma / 2)}{2 \sin (\alpha / 2)}, \\
N= & \left(F_{1}+F_{2}\right) \cos \gamma, \\
\mu= & \frac{\left(F_{1}-F_{2}\right)}{\left(F_{1}+F_{2}\right)} \tan \frac{\gamma}{2} .
\end{aligned}
$$

The average friction coefficient of 0.15 is derived from the measured tension of the radial rod, and the corresponding friction loss is $3.33 \%$ for the hoop cable tension at one joint. The cable tension preslack method was used to simulate construction, and the point-to-point contact element and joint coupling technology were used to simulate the cablesupport joints; the negative temperature was applied to the tensioning points of the hoop cable to study the effects of 

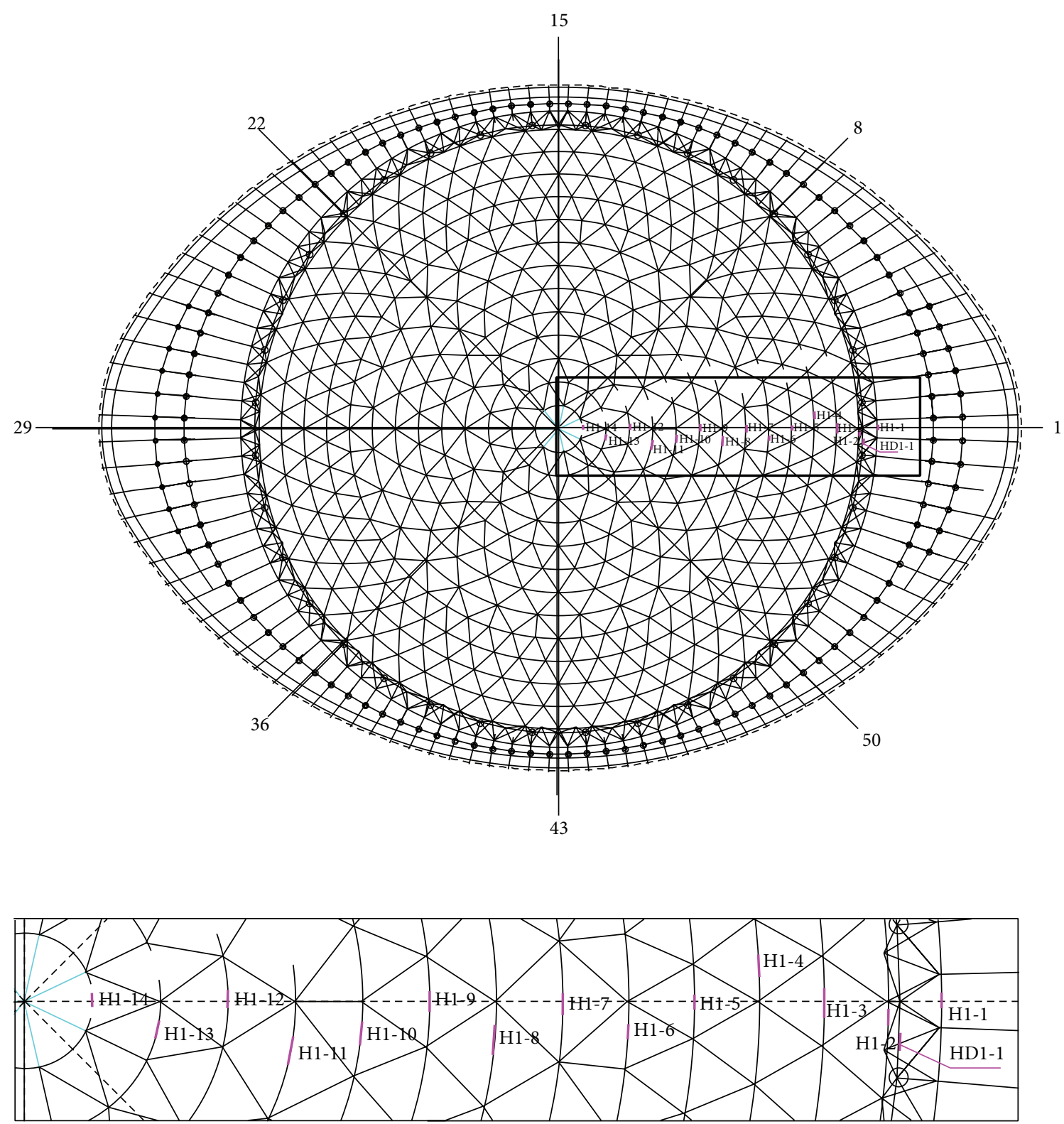

FIGURE 6: Layout of hoop members.

friction. As shown in Table 5, the friction of cable-support joints significantly affects the cable force and improves the tension of the tensioning point. The larger the tension difference between the two steel rods at the same joint, the greater the friction. However, the friction has little effect on the pressure of the strut, which is almost the same as the design value. The friction of the cable-support joint greatly affects the structure and increases the construction difficulty, so this factor must be considered in construction simulations. On the other hand, the design of the cable-support joint must be innovated to reduce the friction as much as possible.

The construction simulation was performed to comprehensively study the effects of the factors mentioned above. The internal forces of the latticed shell members at the tensioned state are shown in Figure 8; the numbers of the members are shown in Figures 6 and 7. As shown in the figure, the joint deviation has little effect on the internal forces. However, the tensioning temperature has a large effect, even if the structure is heated to the design reference temperature, the internal force difference is still large because there is still a temperature difference of $-10^{\circ} \mathrm{C}$ in the latticed shell from the temperature at which it was installed. Other than the two hoop members at the edge of the latticed shell, the temporary supports have little effect, but the stiffness of the temporary supports is difficult to be obtained, which increases the difficulty and uncertainty for the control value of the construction simulation. Therefore, they should be removed before tensioning. The friction of the cable-support 

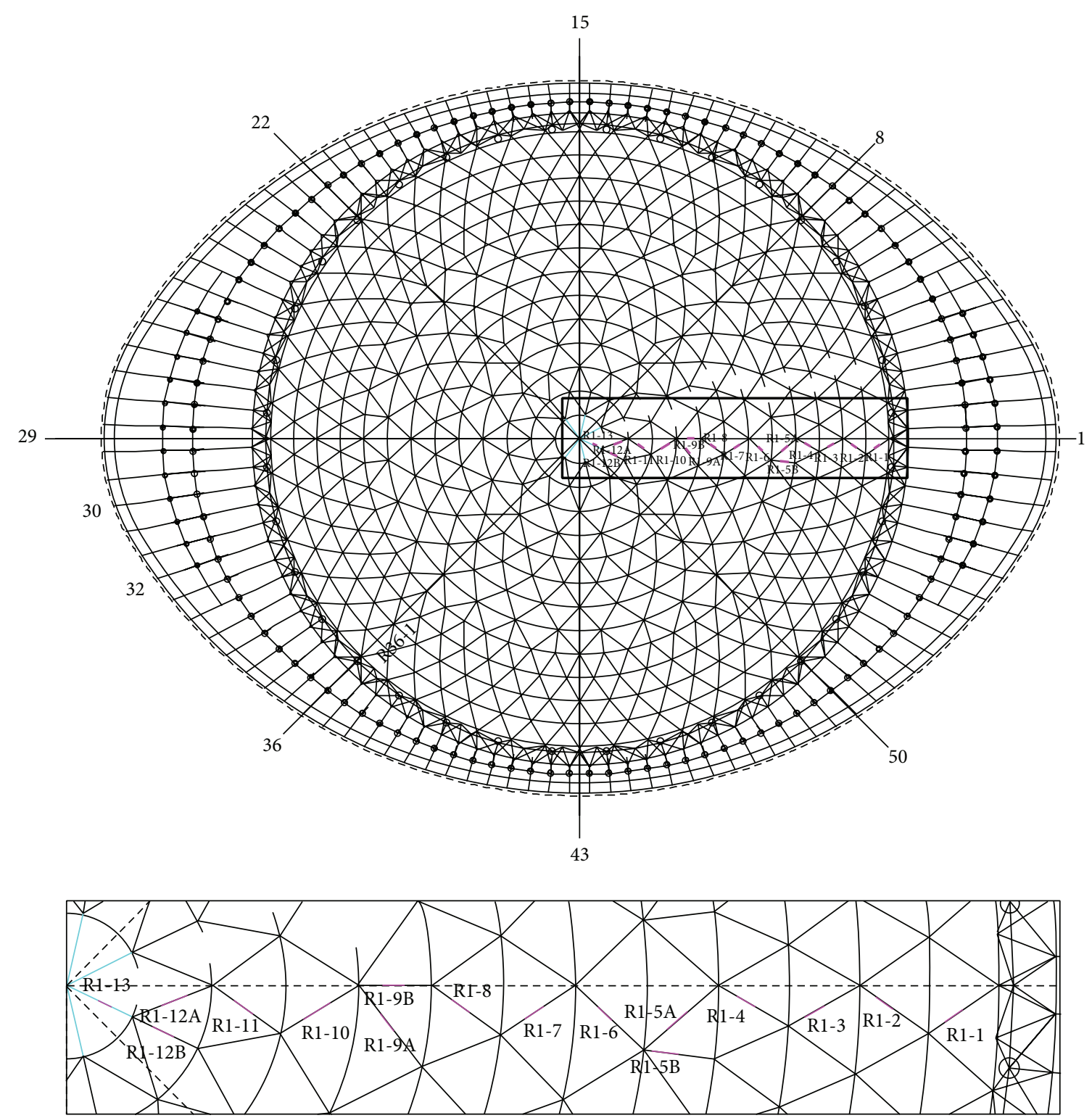

FIGURE 7: Layout of radial members.

joint has a large effect, so the friction must be reduced as much as possible; otherwise, it will not only increase the construction difficulty but also may affect the safety.

The displacements of joints (shown in Figure 9) of the latticed shell are shown in Figure 10. As shown in the figure, except for the joint deviation, the factors all have obvious effects on the joint displacement, particularly on the displacement of the 4 th hoop joints. The temporary supports have little effect on the displacement of the joints inside the 6 th hoop, and the construction temperature and the friction of the cable-support joint obviously affect the displacements of all joints; moreover, these two factors cannot be eliminated in construction and can only be controlled within a certain range, so the effects of these factors must be considered in the construction simulation. The control and acceptance value of the joint deflection must be obtained with the tensioning simulation but not the original design.

6.2. Effects on Load State. To study the effects of these factors on the safety of the suspended dome at the load state, the mechanical characteristics of the structure under design loads are performed, and the results are shown in Figures 11-13. As shown in Figure 11, the friction of the cablesupport joint greatly affects the stress of the radial members of the latticed shell; the maximum difference is $33 \%$, and it has less impact on the hoop members. The joint deviation, construction temperature, and temporary supports have little effect on the internal forces of the structure, which can be neglected in engineering. Temporary supports are removed after the structure is tensioned and before the external loads 


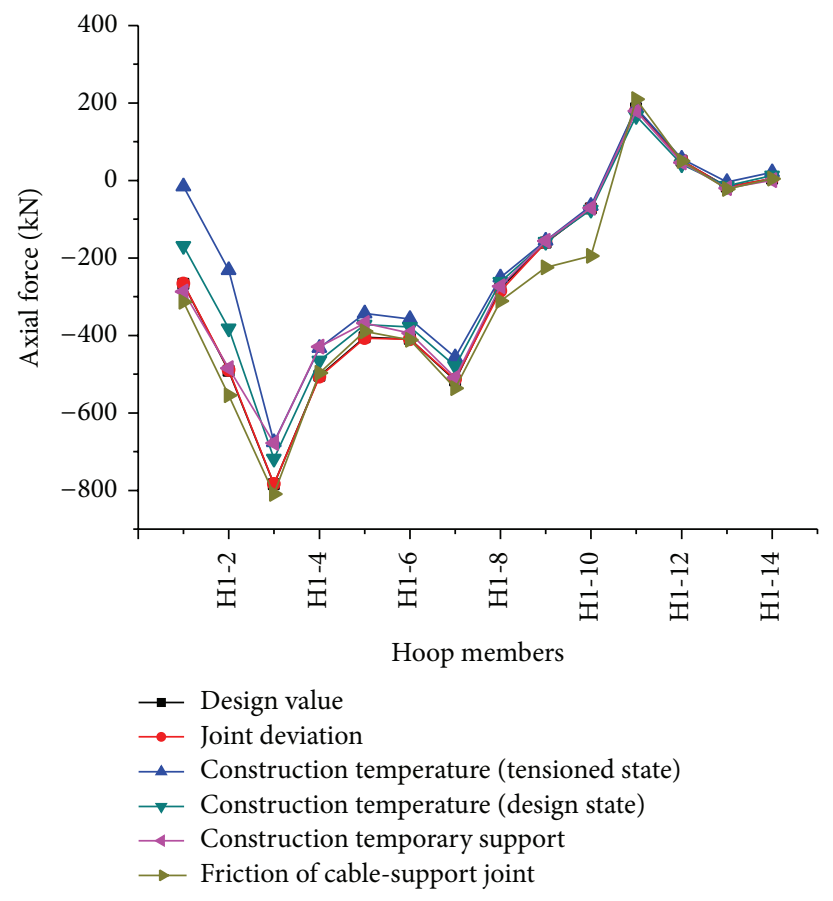

(a) Hoop members

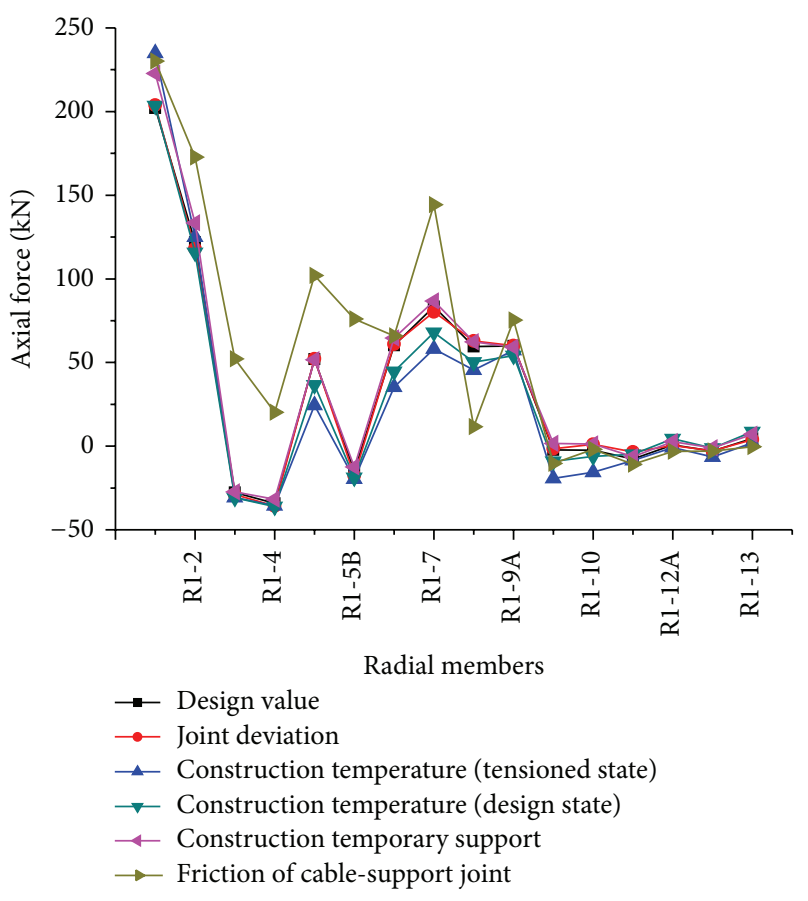

(b) Radial members

Figure 8: Axial force of members on the upper dome.

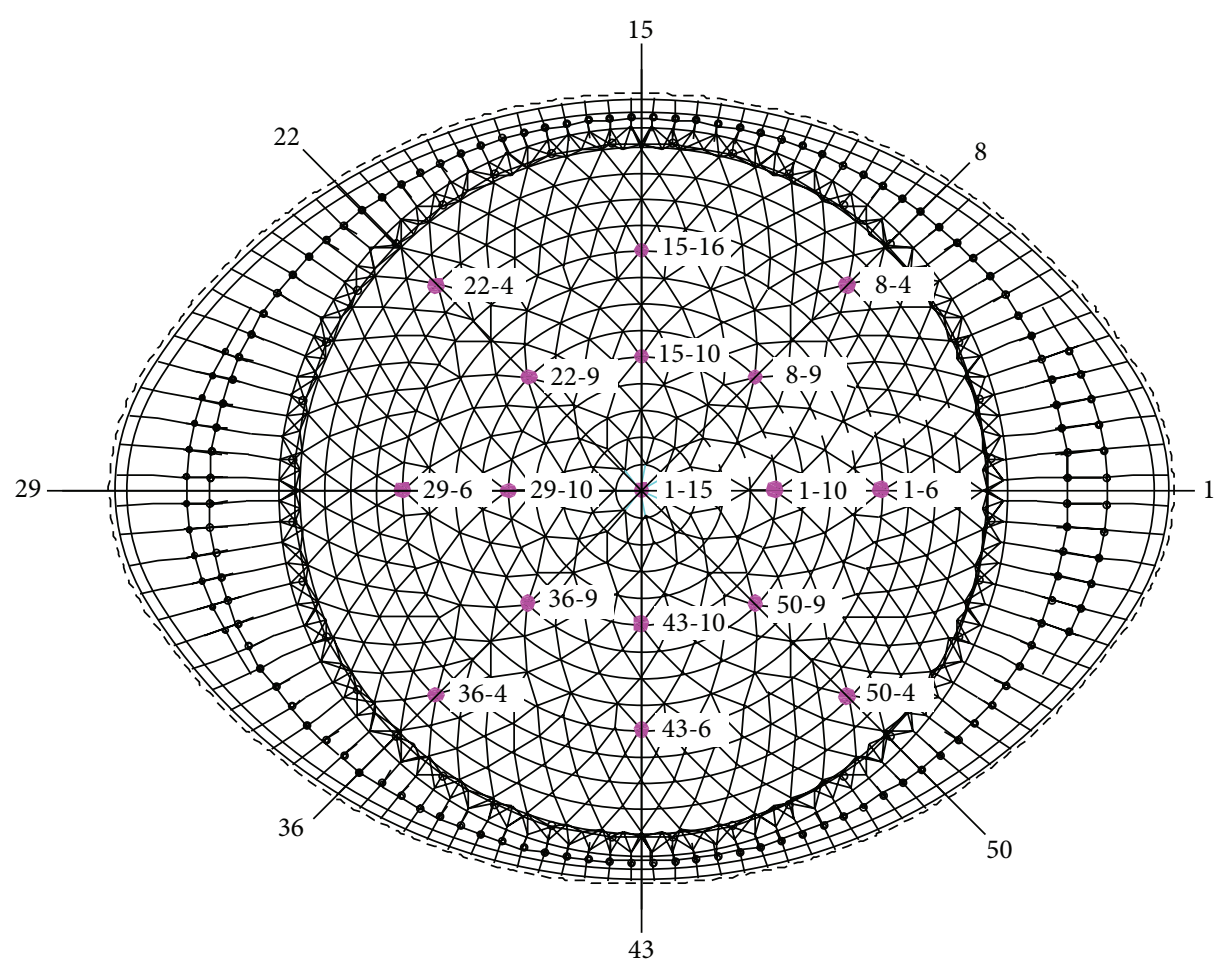

FIGURE 9: Layout of vertical displacement measuring point. 


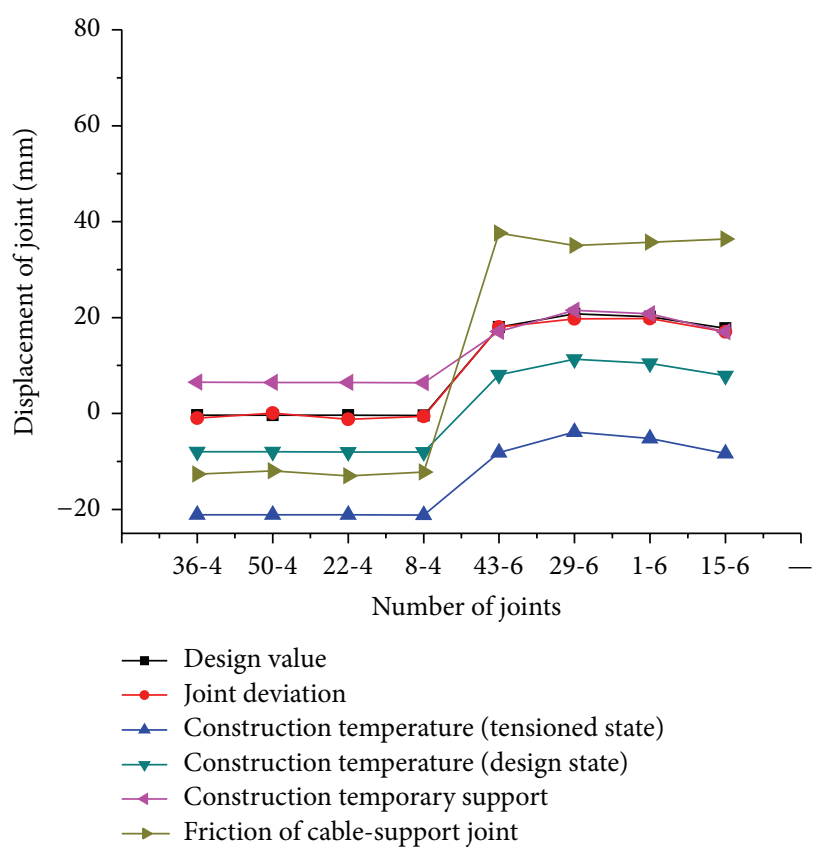

(a) First group of joints

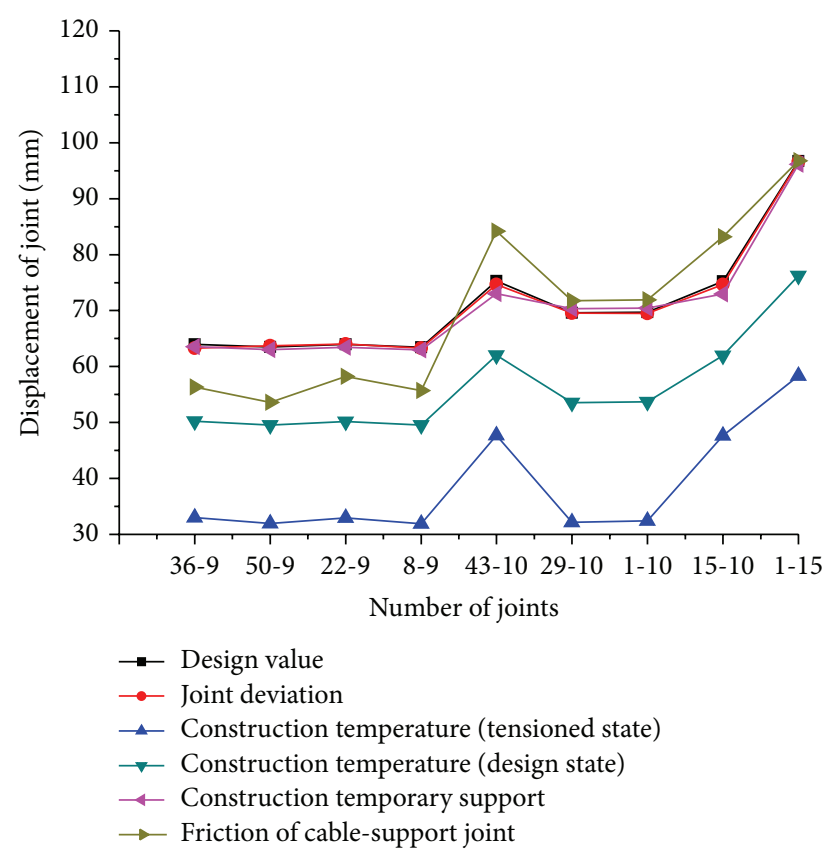

(b) Second group of joints

FIgURE 10: Vertical joint displacement on the upper dome.

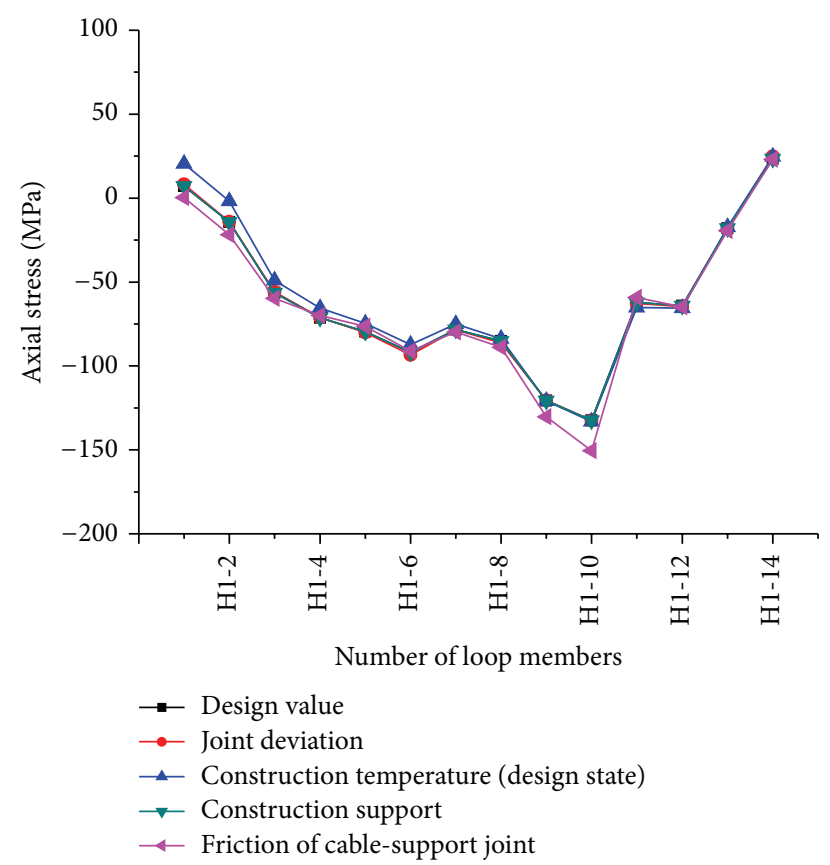

(a) Hoop members

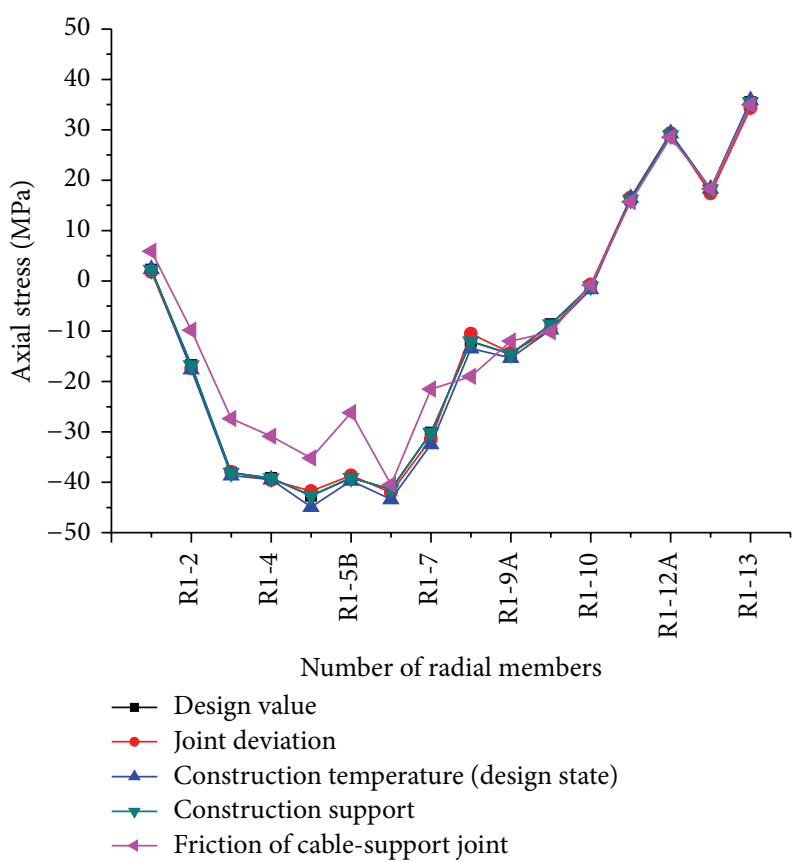

(b) Radial members

FIgUre 11: Axial force of members on the upper dome.

are applied, so the internal force under external loads is the same as the original design internal force.

As shown in Figure 12, the construction temperature and the friction of the cable-support joint obviously affect the deflection of the latticed shell. On the other hand, the location deviation of the joint and construction temporary supports have little effect.

As shown in Figure 13, the location deviation of the joints, construction temperature, and temporary supports have little effect on the force of the cable-support system under external 


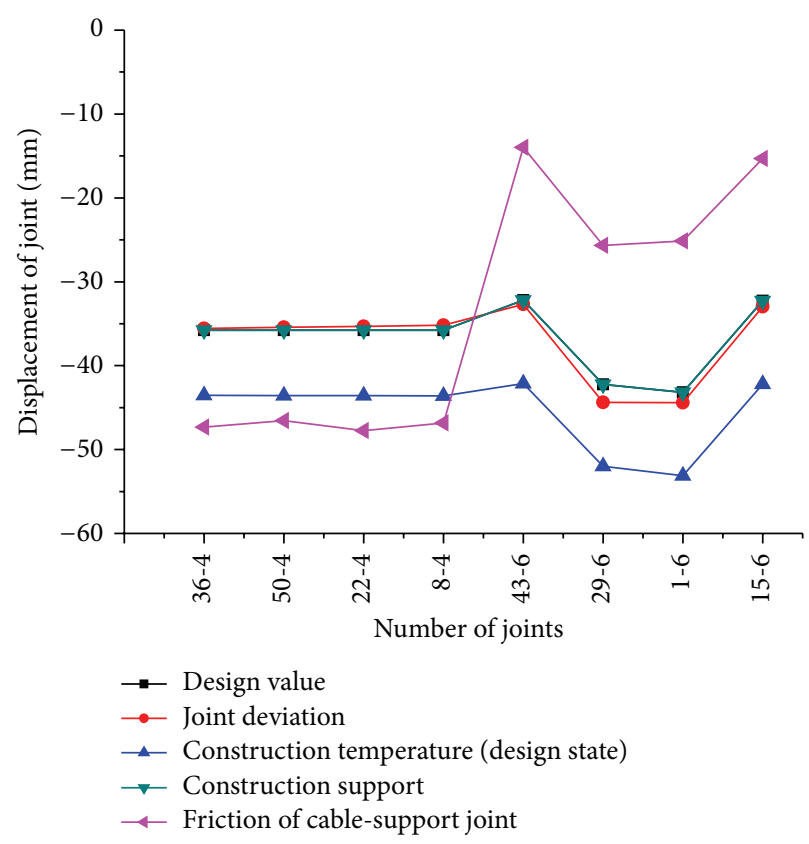

(a) First group of joints

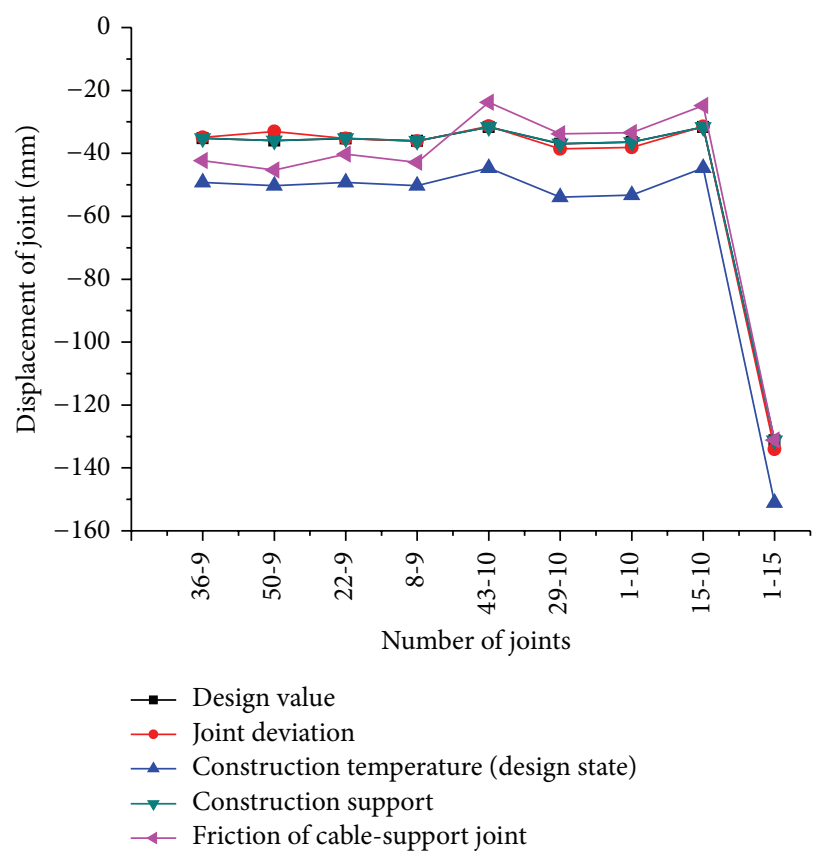

(b) Second group of joints

FIGURE 12: Vertical joint displacement on the upper dome.

loads; however, the friction of the cable-support joints greatly affects the force of the hoop cable and radial steel rod under external loads, especially on the radial steel rods, with a maximum stress difference in the same pair of V-shaped rods of $24 \%$. Therefore, the adverse effect should be considered in the design stage. The friction of the cable-support joint has little effect on the stress of the strut.

\section{Construction Simulation and Tensioning of Case Study}

As shown in Figure 14, the case study was a $93 \mathrm{~m}$ span suspended dome. According to the above analysis, the location deviation of the joint was ignored, the temporary supports were removed before tensioning, and the construction temperature and the friction of the cable-support joint were considered in the construction simulation. The friction coefficient was taken as 0.15 based on field measurements, the latticed shells were installed at $30^{\circ} \mathrm{C}$, the tensioning was at $0^{\circ} \mathrm{C}$, and the design reference temperature is $20^{\circ} \mathrm{C}$. The construction simulation of the large-span suspended dome was performed, and the structure was tensioned according to the results of the simulation, and the simulation results were compared with the results measured on site. As shown in Figure 1, 16 tensioning points of hoop cable were set, and the tensioning process was divided into three stages: the 5 hoop cables were tensioned to $70 \%, 90 \%$, and $100 \%$ of the simulation value in three stages. Each cable was tensioned to $70 \%$ in the first stage in sequence $\mathrm{A} \rightarrow \mathrm{B} \rightarrow \mathrm{C} \rightarrow \mathrm{D} \rightarrow$ $\mathrm{E}$, and then each cable was overtensioned to $105 \%$ and then unloaded to $90 \%$ in the second stage in the sequence
$\mathrm{A} \rightarrow \mathrm{B} \rightarrow \mathrm{C} \rightarrow \mathrm{D} \rightarrow \mathrm{E}$. Finally, each cable was overtensioned to $115 \%$ and then unloaded to $110 \%$ in the third stage in the sequence $\mathrm{E} \rightarrow \mathrm{D} \rightarrow \mathrm{C} \rightarrow \mathrm{B} \rightarrow \mathrm{A}$. To tension synchronously, each tensioning step was subdivided into 4 to 10 substeps. The jacking was done synchronously in each substep and then jacking was stopped after each substep finished before measuring the elongation of the cable. If the elongations at the tensioning points were different, the tensioning point with the smallest elongation was tensioned to make up this difference and then the tensioning points were tensioned simultaneously.

7.1. Layout of the Measuring Point. According to the internal force of the members and the failure mode of the integral instability in the model test, the vibratory strain gauges were set in the locations where the internal forces were large to measure the axial forces of members. The layout of the measuring points is shown in Figure 15. The sensors were set on the upper and lower surfaces of the tube near the joints, and the average value was used to calculate the axial forces of the members.

The total station was used to measure the vertical joint displacements, and each joint was measured at each tensioning stage. Only the representative measuring points shown in Figure 16 were analyzed in this paper; because of the symmetry of the structure and tensioning points, these measuring points show the maximum displacement of the structure and reflect the change law of displacement.

7.2. Hoop Cable Tension. The data for the processes of $70 \%-90 \%$ and $70 \%-110 \%$ were compared and analyzed in 


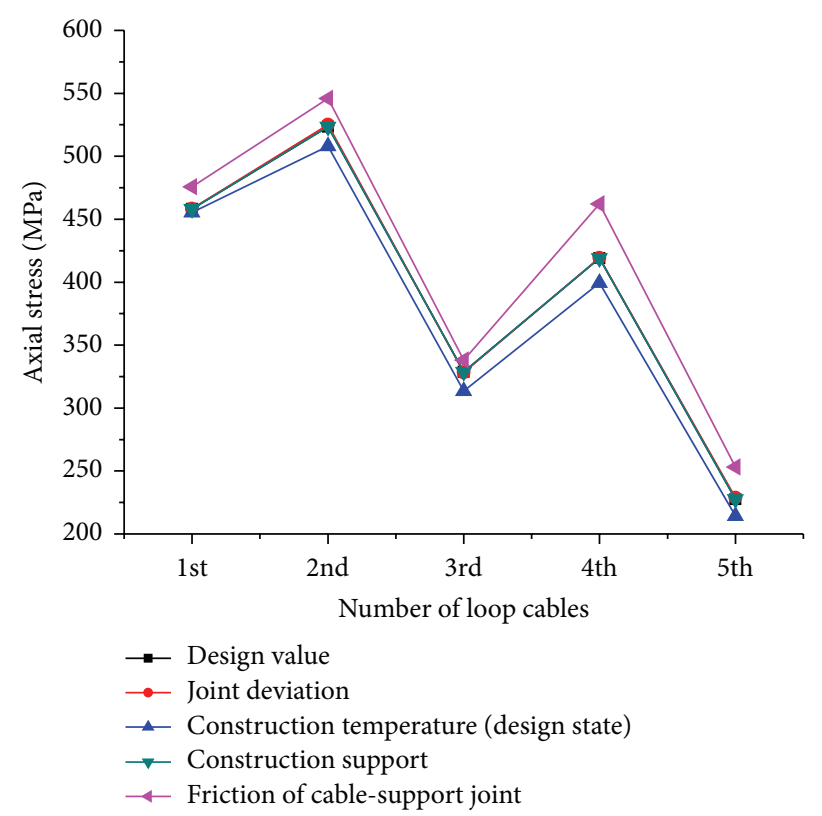

(a) Hoop cable

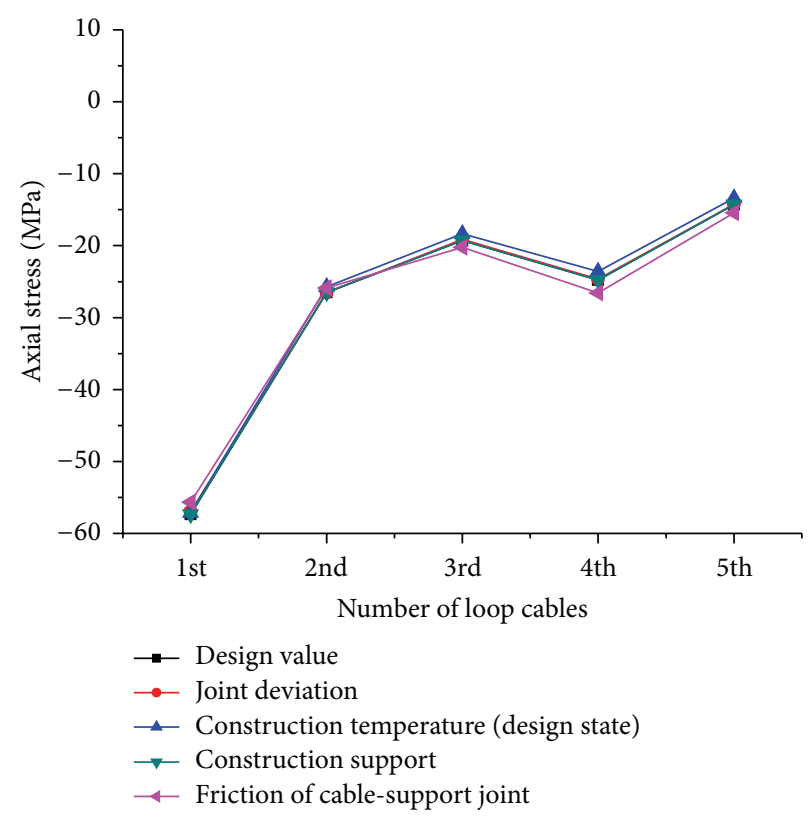

(b) Strut

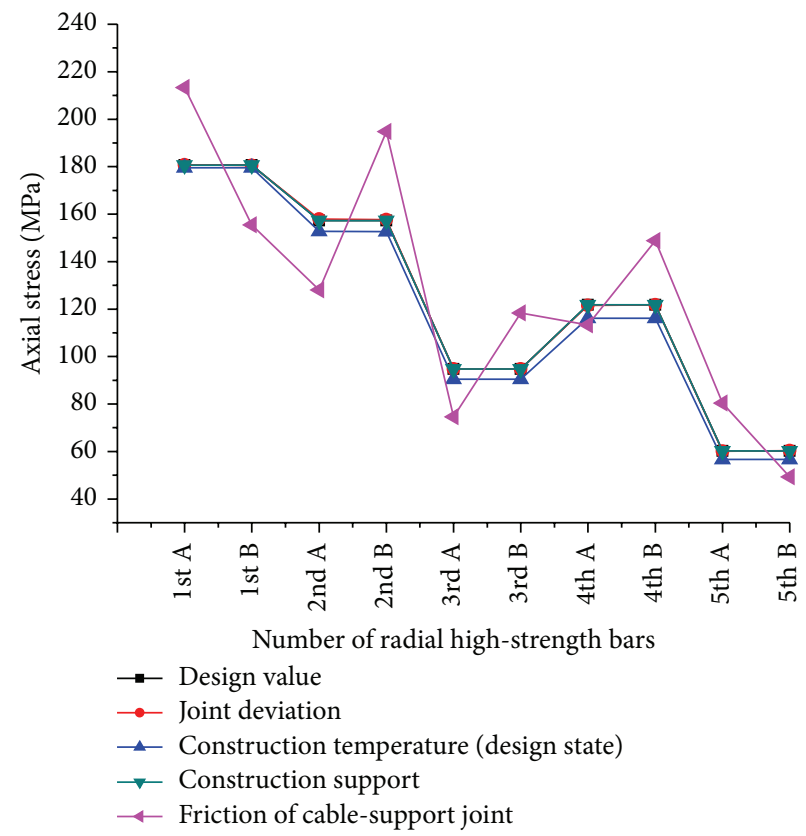

(c) Radial steel rod

FIGURE 13: Axial stress of hoop cables.

this paper. As shown in Figure 17, the discreteness of the measured values of the cable force in the $70 \%-110 \%$ stage was smaller than that in the 70\%-90\% process except for cable A. The measured value of cable A had the largest difference with the simulation value, and the measured value of cable $B$ was slightly lower than the simulation value; the measured and simulation values of cables $\mathrm{C}, \mathrm{D}$, and $\mathrm{E}$ were very close. With increasing tension, the hoop cable can overcome the friction of the cable-support joint more easily, but with increasing cable force, applying the cable force is increasingly difficult, causing the measured values of cables $\mathrm{A}$ and $\mathrm{B}$, which have large cable forces, to be lower than the simulation values. In general, the measured values and simulation values were close.

7.3. Tension of Radial Steel Rod. The tension difference between a pair of steel rods at the same cable-support joint is large because of the friction of the cable-support joint. As shown in Figure 18, the tensions of $\mathrm{A} 2$ and $\mathrm{A} 3$ in the $70 \%-90 \%$ stage were largely different from the simulation 


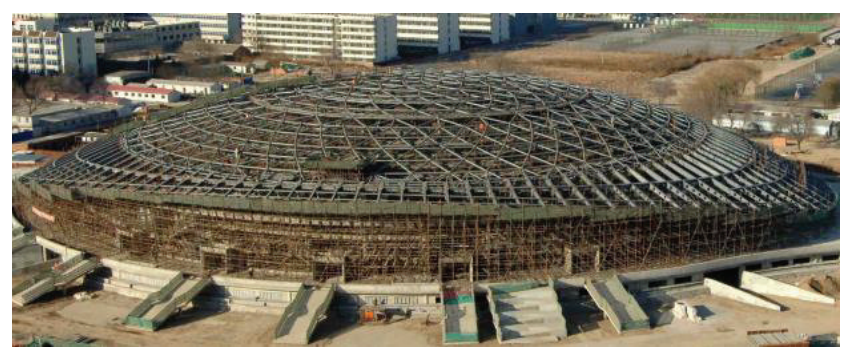

(a) Outside picture

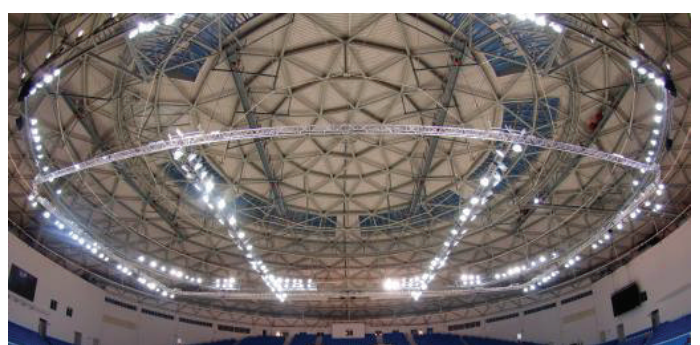

(b) Inside picture

FIGURE 14: Suspended dome structure project.

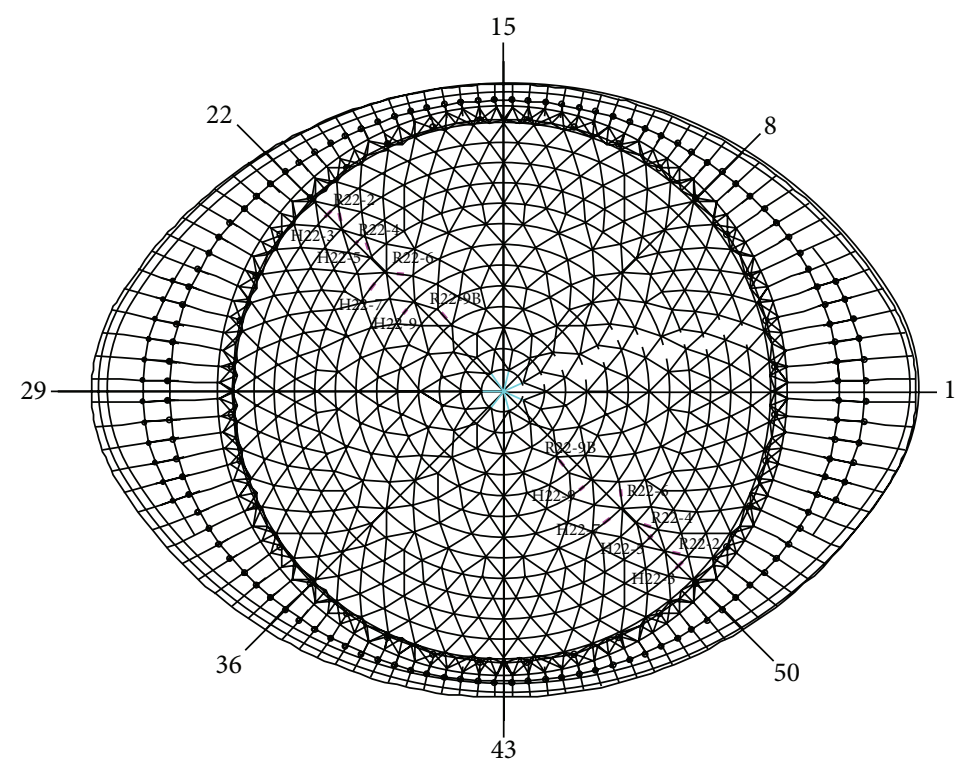

(a) Layout

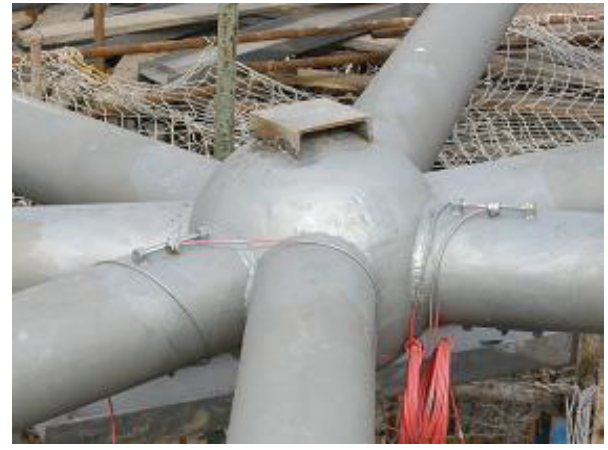

(b) Picture

FIGURE 15: Layout of the vibrating wire sensor.

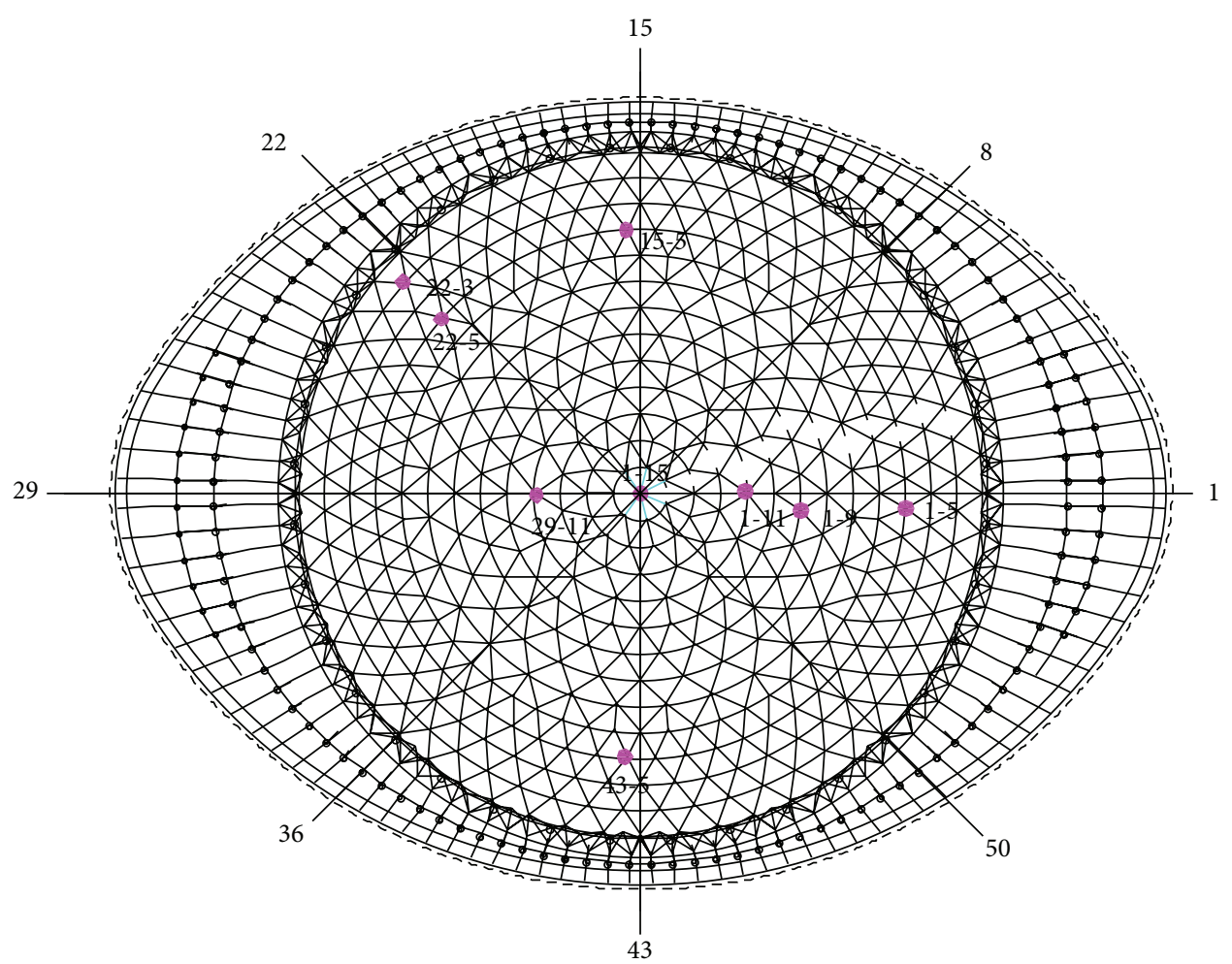

FIGURE 16: Layout of the vertical displacement measuring points. 


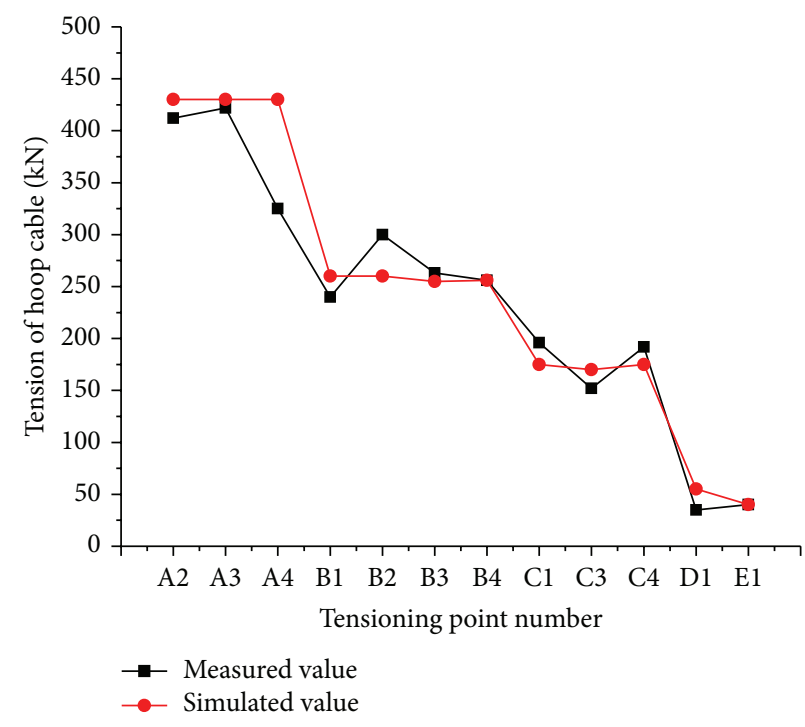

(a) $70 \%-90 \%$ stage

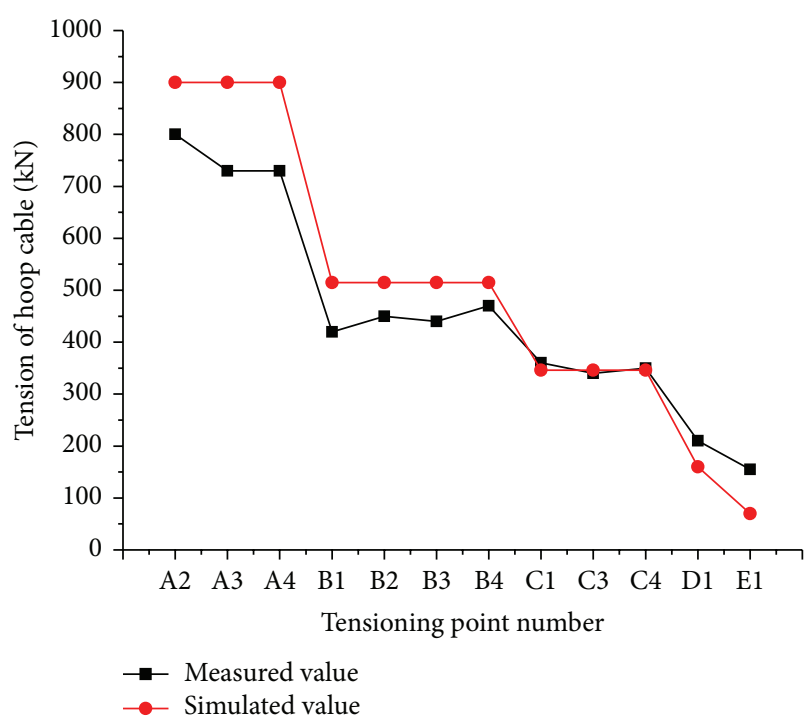

(b) $70 \%-110 \%$ stage

FIGURE 17: Tension increment of the hoop cable.

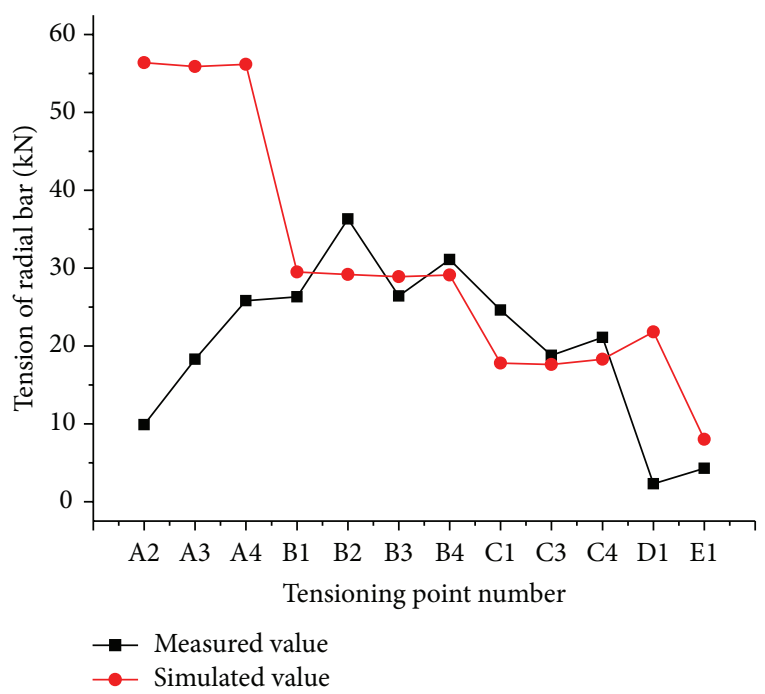

(a) Rod inside the tensioning point

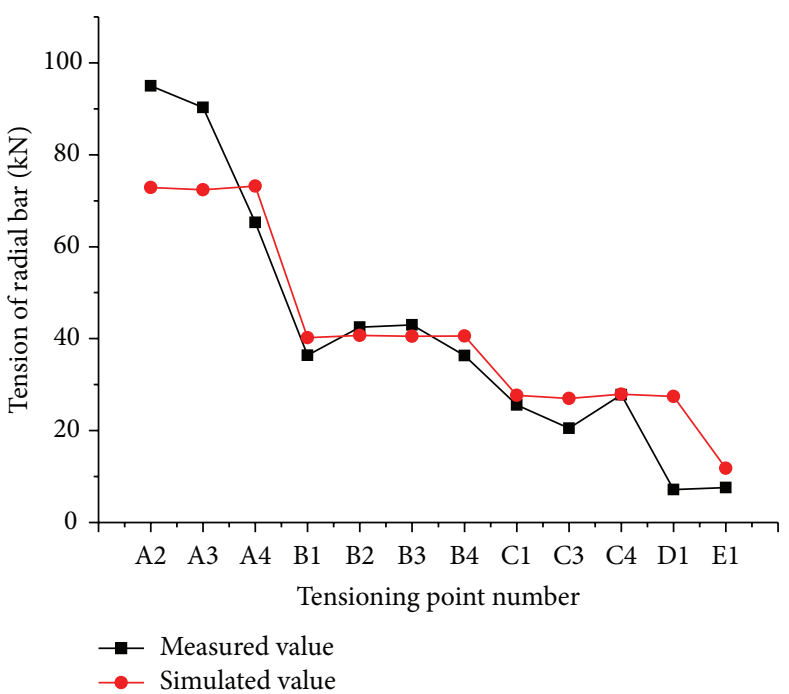

(b) Rod outside the tensioning point

FIGURE 18: Tension increment of the radial bar in the 70\%-90\% stage.

values, showing that the frictions of the cable-support joints at these two tensioning points were bigger than the values used in the construction simulation. As shown in Figure 19, with increasing tension, the friction at these two tensioning points decreases, and the tensions of each pair of steel rods are close, showing that as the tensioning proceeds, the friction of the cable-support joint is effectively overcome. The tension of the steel rod outside of the tensioning point is larger than that inside and is closer to the simulated value. In general, the discreteness of the steel rod tension is large, so it should not be used alone as the acceptance criteria. Instead, the resultant force of the pair of rods should be compared with that of the simulated value, which is used as the auxiliary parameter of the acceptance.
7.4. Vertical Displacement. The vertical joint displacements in the latticed shell at the $70 \%$ and $110 \%$ tensioning state are compared in Figure 20. As shown in the figure, the measured vertical displacements align well with the simulated values. Therefore, the construction met the design requirements taking the displacement as the standard.

7.5. Axial Force of Struts. As shown in Figure 21, the simulated axial force of the struts in the $70 \%-90 \%$ stage aligns well with the measured values for most of the struts. The simulated values were closer to the measured value in the $70 \%-110 \%$ process, which clearly shows that the tension is larger, and the simulated value is closer to the measured value. 


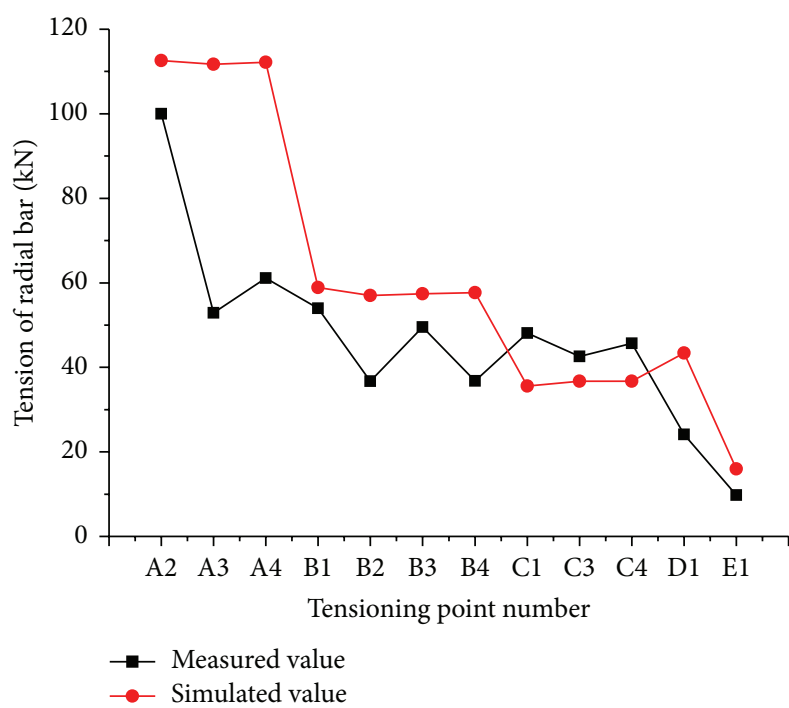

(a) Rod inside the tensioning point

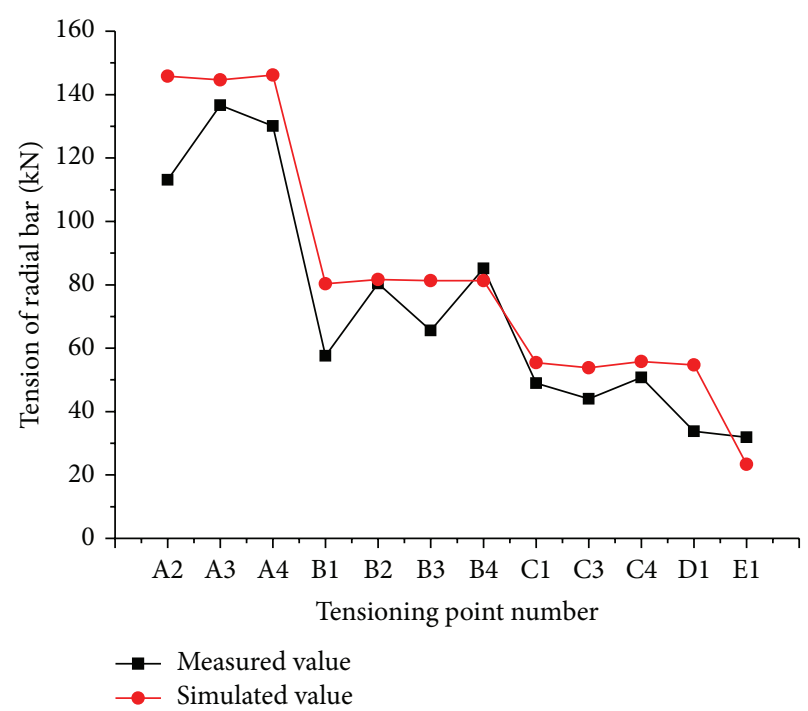

(b) Rod outside the tensioning point

FIgURE 19: Tension increment of the radial bar in the $70 \%-110 \%$ process.

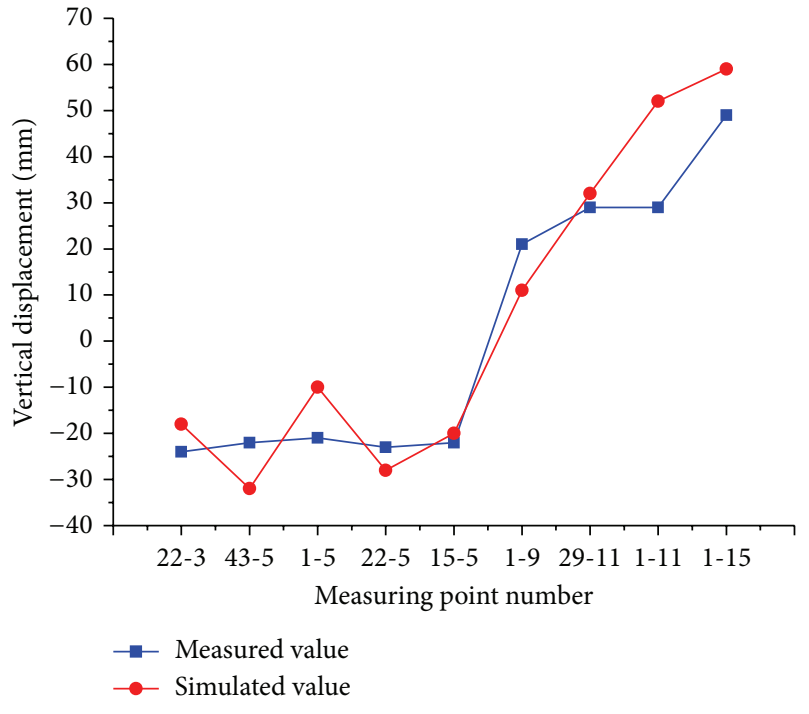

(a) $70 \%$ tensioning state

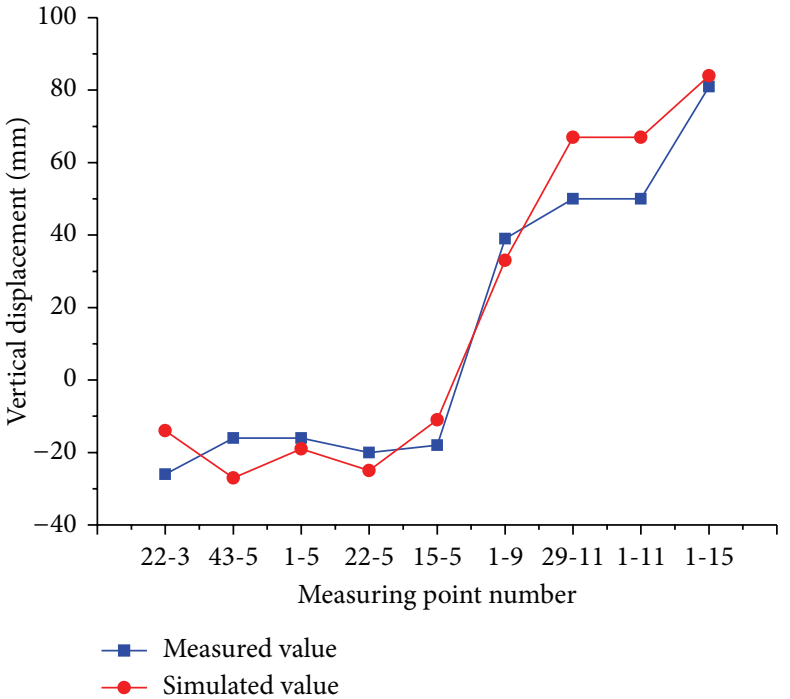

(b) $110 \%$ tensioning state

FIGURE 20: Joint displacement.

7.6. Axial Force of Members in the Latticed Shell. As shown in Figures 22 and 23, the simulated internal force of the members in the latticed shell match the measured values better in the $70 \%-110 \%$ stage than in the $70 \%-90 \%$ process. For members H50-3 and H50-5, the measured axial force was smaller than the simulated value, which is beneficial for the structure. The tension is larger and the measured value is closer to the simulated value, which shows that as the tension increases, the effects of some uncertain factors including friction, support stiffness, and joint stiffness are gradually reduced. The simulated axial forces of the hoop members meet the measured values better than that of the radial members because the internal forces of the radial members is small and although the absolute difference is not large, the relative difference is.

\section{Conclusions}

Based on the friction element, the joint coupling technology, and the local cooling, the cable tension preslack method for the construction simulation of prestressed steel structure was proposed in this paper, and the effects of the location deviation of joints, construction temperature, construction temporary supports, and the friction of cable-support joints on the simulation results were analyzed. The method was used for engineering a large-span suspended dome, and 


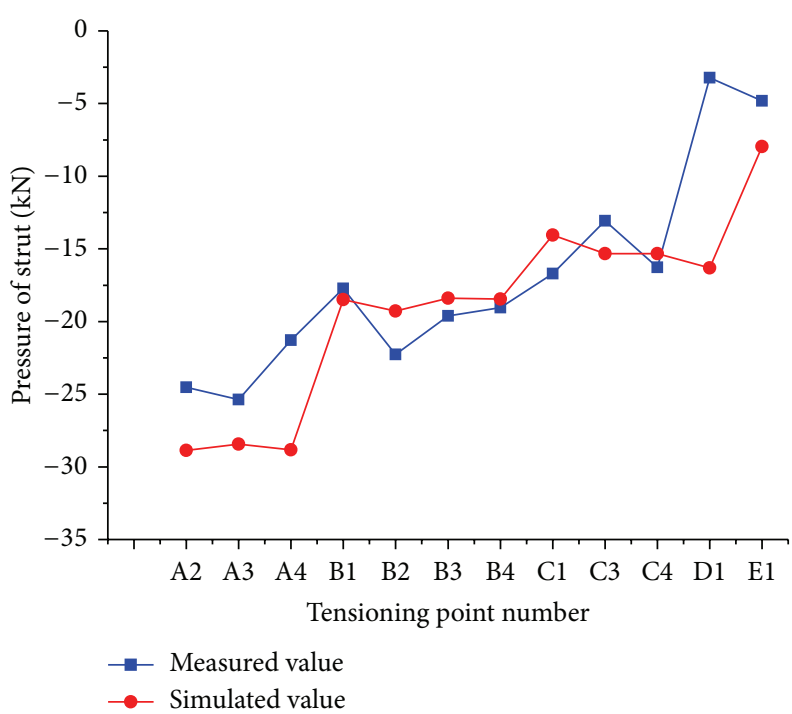

(a) $70 \%-90 \%$ stage

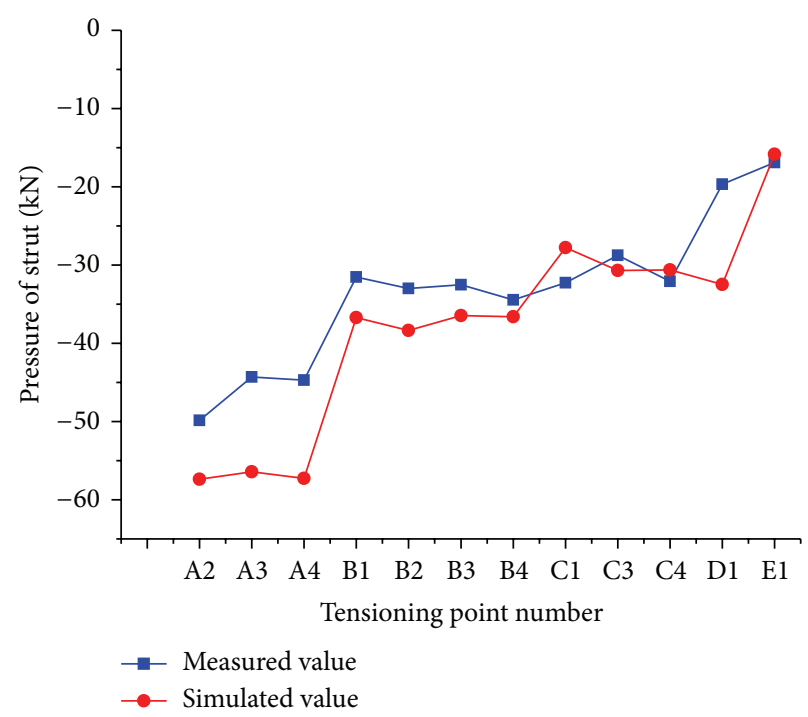

(b) $70 \%-110 \%$ stage

FIGURE 21: Pressure increment of strut.

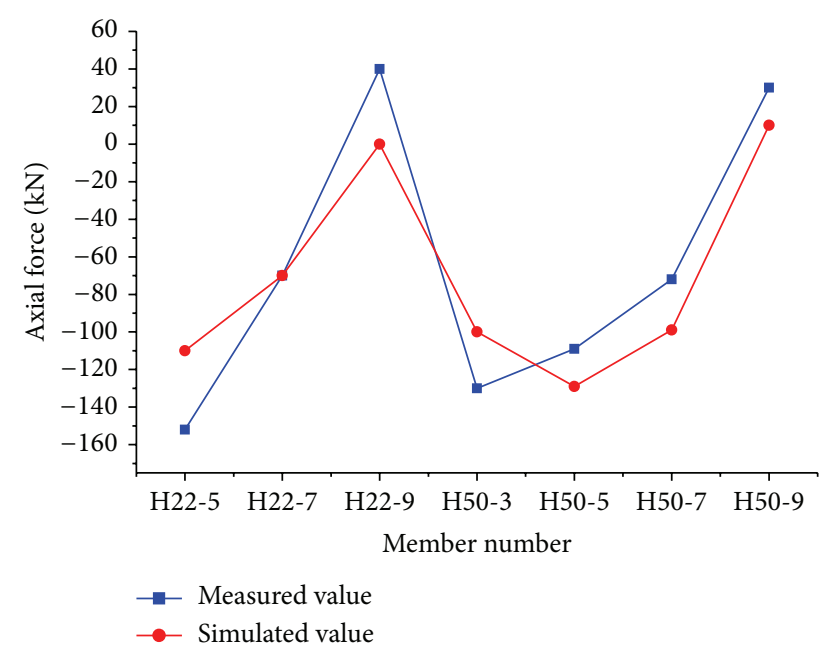

(a) Hoop members

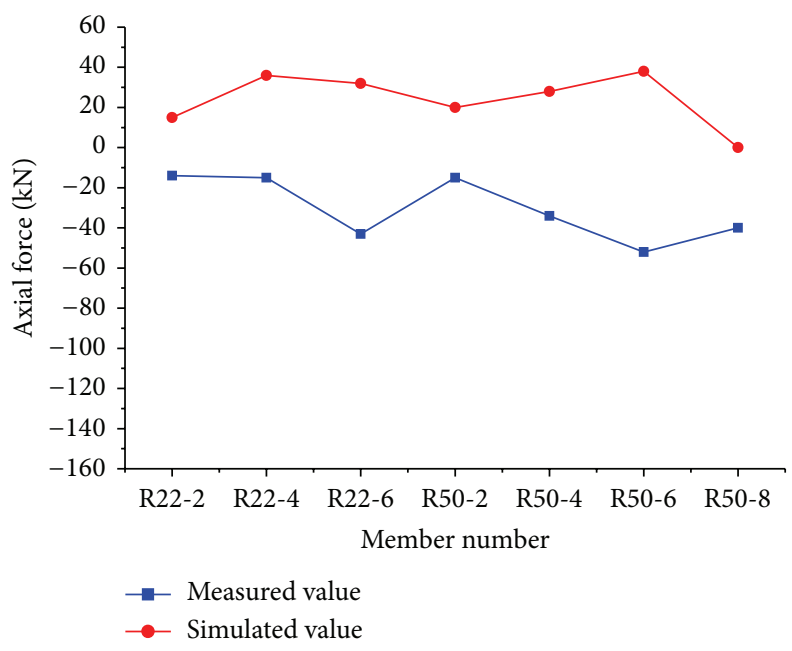

(b) Radial members

FIGURE 22: Force increment of the members in the $70 \%-90 \%$ process.

the simulation results were compared with measured results to verify the simulation method and obtain the following conclusions.

(1) The construction temperature and the friction of the cable-support joint obviously affect the tensioning value of the suspended dome, so these factors must be considered in the construction simulation.

(2) The construction of temporary supports results in many uncertain factors, and accurate tension control is difficult to be obtained in the construction simulation, so they should be removed before tensioning.

(3) The construction temperature, construction temporary supports, and friction of the cable-support joints all affect the internal force and deflection of the simulation, but the effect on the mechanical characteristics of the structure at the load state is not so obvious. The force should be mainly controlled while the control of deflection is secondary in tensioning construction. The measured deflection should be compared with the simulated deflection considering various factors, but not with the original design value.

(4) The cable tension preslack method proposed in this paper can include various factors to provide an accurate simulation analysis and verification method for the construction of a prestressed steel structure. Before the construction, the tensioning process of a case study was previewed, which provided important 


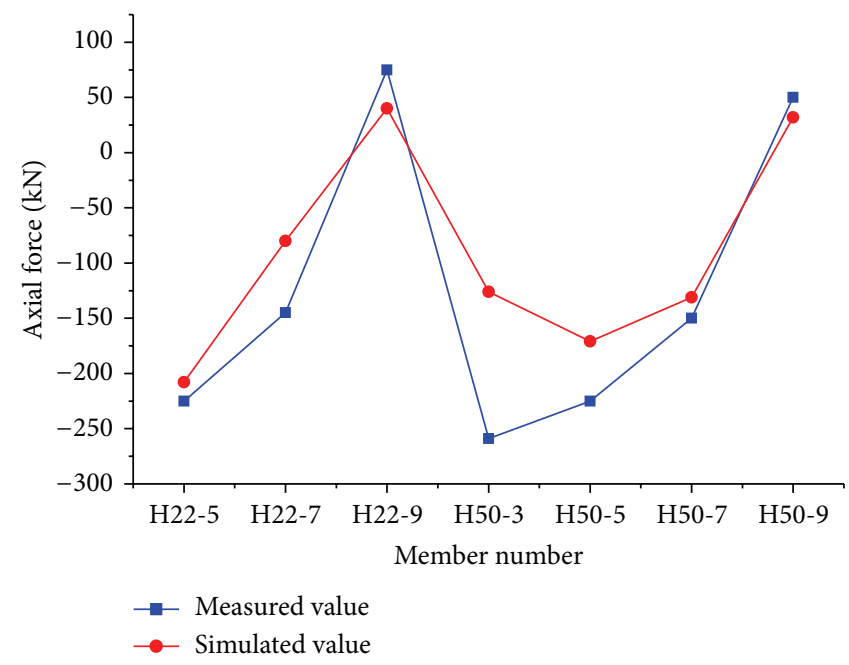

(a) Hoop members

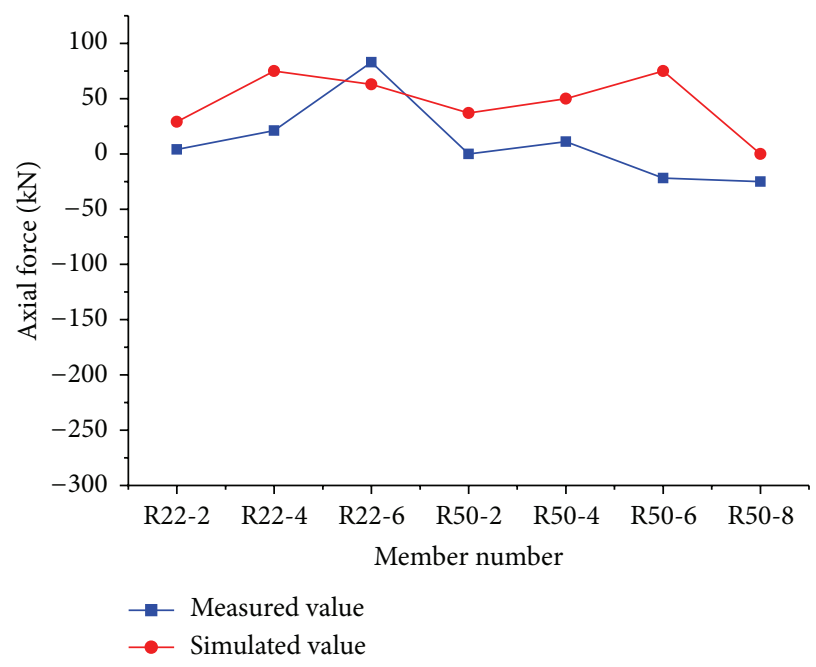

(b) Radial members

FIGURE 23: Force increment of the members in the $70 \%-110 \%$ process.

data for the selection of the tensioning scheme and construction acceptance standards.

\section{Conflict of Interests}

The authors declare that there is no conflict of interests regarding the publication of this paper.

\section{Acknowledgment}

Financial support for this study was from the National Natural Science Foundation of China (Grant no. 51248009).

\section{References}

[1] M. Kawaguchi, M. Abe, and I. Tatemichi, "Design, tests and realization of 'suspend dome' system," Journal of the IASS, vol. 40, no. 131, pp. 179-192, 1999.

[2] M. Kawaguchi, M. Abe, T. Hatato et al., "Structural tests on the 'suspen-dome' system," in Proceedings of the IASS-ASCE International Symposium, pp. 383-392, April 1994.

[3] J. A. Lozano-Galant, I. Payá-Zaforteza, D. Xu, and J. Turmo, "Analysis of the construction process of cable-stayed bridges built on temporary supports," Engineering Structures, vol. 40, pp. 95-106, 2012.

[4] J. A. Lozano-Galant, X. Dong, I. Payá-Zaforteza, and J. Turmo, "Direct simulation of the tensioning process of cable-stayed bridges," Computers \& Structures, vol. 121, pp. 64-75, 2013.

[5] Y. M. Li, Y. G. Zhang, and Q. S. Yang, "Determination of cable forces during construction for cable-supported lattice shells," Journal of Building Structures, vol. 25, no. 4, pp. 76-81, 2004.

[6] X. F. Yuan and S. L. Dong, "Inverse analysis of construction process of cable dome," Journal of Building Structures, vol. 22, no. 2, pp. 75-79, 2001.

[7] G. J. Zhang, J. Q. Ge, J. Qin, Z. Q. Wang, and J. R. Wang, "Simulating analysis of prestressed construction process of the suspend dome of badminton gymnasium for 2008 Olympic
Games," Journal of Building Structures, vol. 28, no. 6, pp. 31-38, 2008.

[8] X. Zhuo, "Tension compensation analysis method and application in prestressed space grid structures tension construction," China Civil Engineering Journal, vol. 37, no. 4, pp. 38-45, 2004.

[9] Z. Q. Wang, J. Qin, and G. L. Li, “Treatment of hoop cable and support condition and experimental study on the annular ellipse suspen-dome," Spatial Structures, vol. 12, no. 3, pp. 12-17, 2006.

[10] Z. Q. Wang, J. Qin, G. L. Li, and X. L. Chen, "Experiment study on two type of cable arrangements of the double ellipse suspend-dome," Industrial Buildings, vol. 36, pp. 477-480, 2004.

[11] A. L. Zhang, D. M. Huang, and C. C. Zhang, "All course analysis of suspendome construction,” Industrial Buildings, vol. 37, no. 4, pp. 55-59, 2007.

[12] J. M. Guo, "Theoretical and experimental study on tensioning of suspend dome," China Civil Engineering Journal, vol. 44, no. 2, pp. 65-71, 2011.

[13] G. B. Nie, X. D. Zhi, F. Fan, and S. Z. Shen, "Study of the tension formation and static test of a suspendome for Dalian Gymnasium," China Civil Engineering Journal, vol. 45, no. 2, pp. $1-10,2012$.

[14] GB50017-2003. Code for Design of Steel Structures, vol. 21, China Planning Press, 2003. 

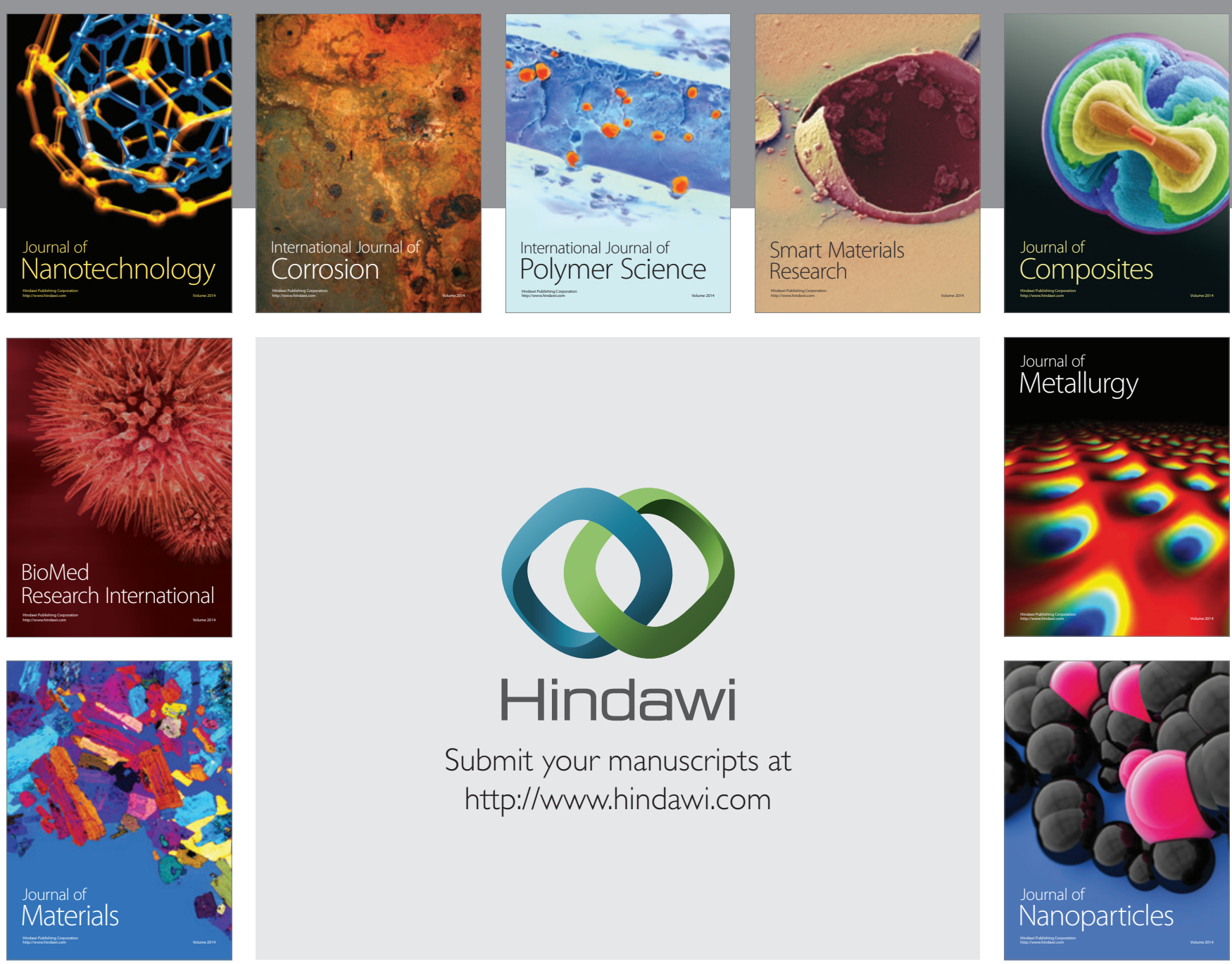

Submit your manuscripts at http://www.hindawi.com
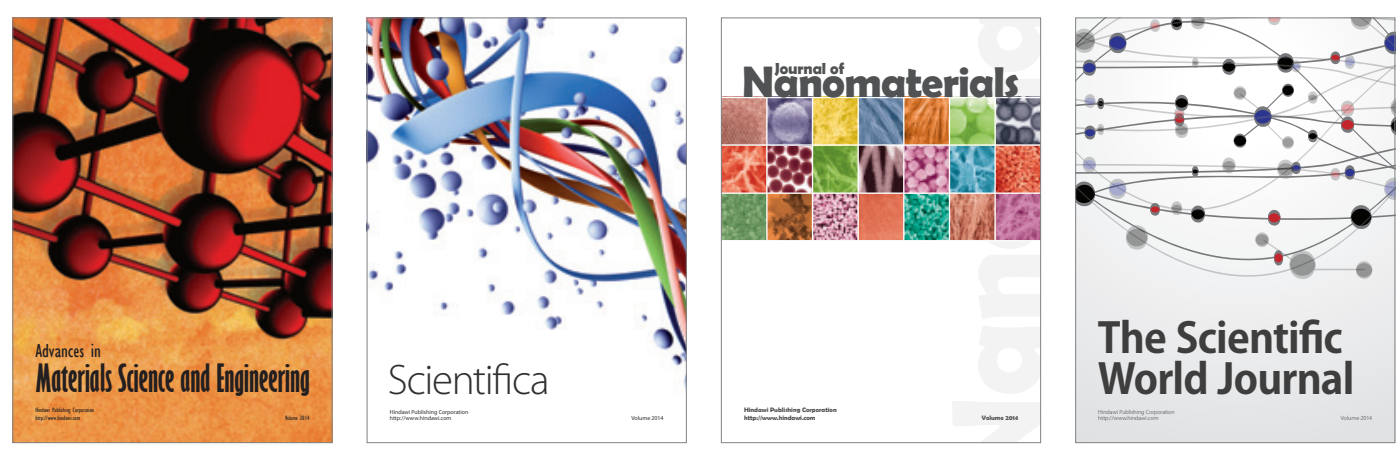

\section{The Scientific World Journal}
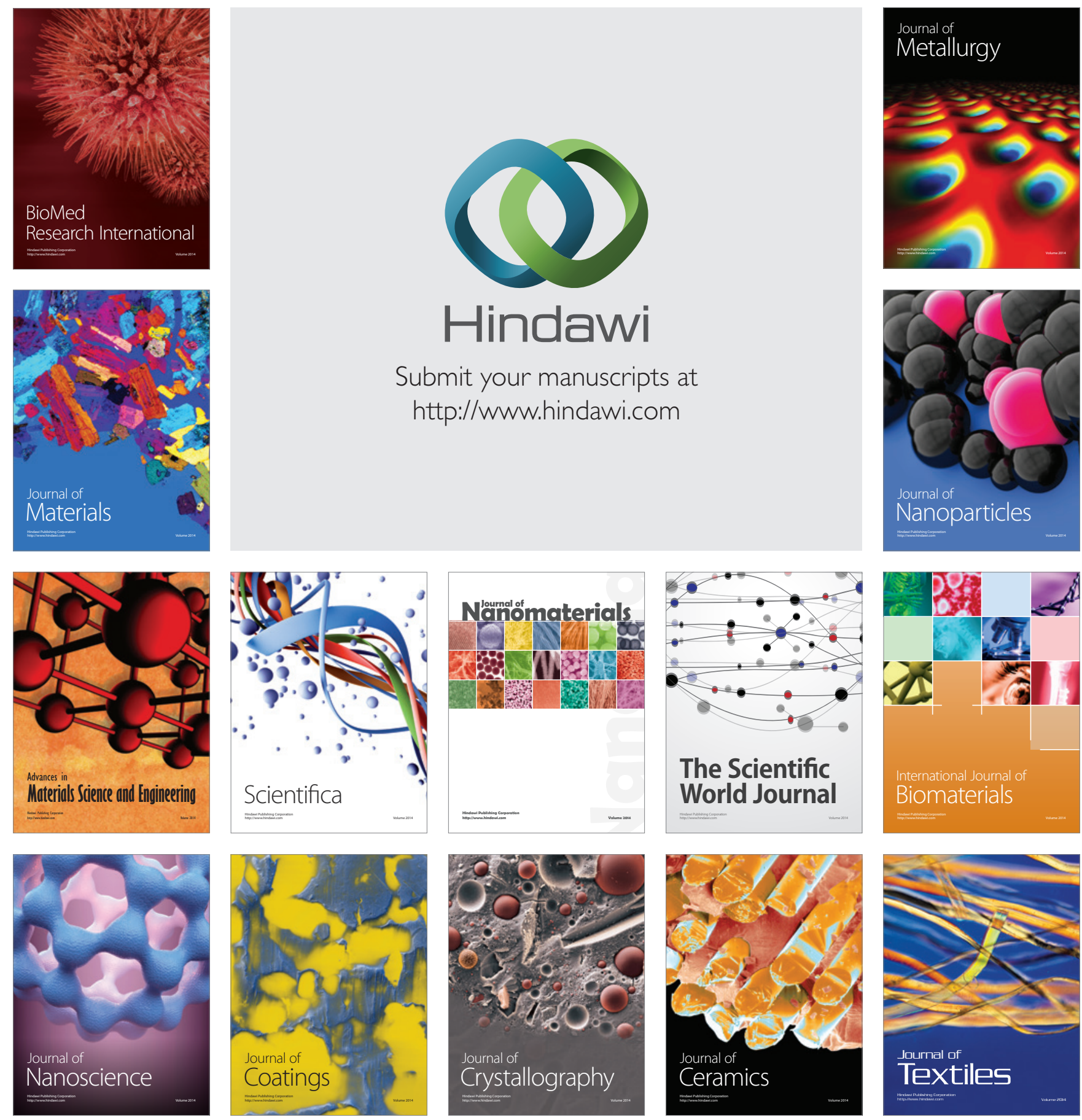